\title{
SPARC promotes leukemic cell growth and predicts acute myeloid leukemia outcome
}

\author{
Houda Alachkar,,$^{1,2}$ Ramasamy Santhanam,1,2 Kati Maharry, ${ }^{2,3}$ Klaus H. Metzeler, ${ }^{2}$ \\ Xiaomeng Huang, ${ }^{1,2}$ Jessica Kohlschmidt, ${ }^{2,3}$ Jason $\mathrm{H}$. Mendler, ${ }^{1,2}$ Juliana M. Benito, ${ }^{4}$ \\ Christopher Hickey, ${ }^{1,2}$ Paolo Neviani, 2,5 Adrienne M. Dorrance,,1,2 Mirela Anghelina, ${ }^{1,2}$ \\ Jihane Khalife, ${ }^{1,2}$ Somayeh S. Tarighat, ${ }^{1,2}$ Stefano Volinia, ${ }^{5}$ Susan P. Whitman, ${ }^{2,5}$ Peter Paschka, ${ }^{2}$ \\ Pia Hoellerbauer, ${ }^{1,2}$ Yue-Zhong Wu, ${ }^{1,2}$ Lina Han, ${ }^{4}$ Brad N. Bolon, ${ }^{6}$ William Blum, ${ }^{1,2}$ \\ Krzysztof Mrózek, ${ }^{2}$ Andrew J. Carroll,7 Danilo Perrotti, ${ }^{2,5}$ Michael Andreeff, ${ }^{4}$ Michael A. Caligiuri, ${ }^{1,2}$ \\ Marina Konopleva, ${ }^{4}$ Ramiro Garzon,, ${ }^{1,2}$ Clara D. Bloomfield,,1,2 and Guido Marcucci'1,2,5 \\ ${ }^{1}$ Division of Hematology, Department of Medicine, and ${ }^{2}$ Comprehensive Cancer Center, The Ohio State University (OSU), Columbus, Ohio, USA. \\ ${ }^{3}$ Alliance for Clinical Trials in Oncology Statistics and Data Center, Mayo Clinic, Rochester, Minnesota, USA. ${ }^{4}$ Department of Leukemia, \\ The University of Texas MD Anderson Cancer Center, Houston, Texas, USA. ${ }^{5}$ Department of Molecular Virology, Immunology and Cancer Genetics, \\ and ${ }^{6}$ Comparative Pathology and Mouse Phenotyping Shared Resource, Comprehensive Cancer Center, OSU, Columbus, Ohio, USA. \\ ${ }^{7}$ Department of Genetics, University of Alabama at Birmingham, Birmingham, Alabama, USA.
}

\begin{abstract}
Aberrant expression of the secreted protein, acidic, cysteine-rich (osteonectin) (SPARC) gene, which encodes a matricellular protein that participates in normal tissue remodeling, is associated with a variety of diseases including cancer, but the contribution of SPARC to malignant growth remains controversial. We previously reported that SPARC was among the most upregulated genes in cytogenetically normal acute myeloid leukemia (CN-AML) patients with gene-expression profiles predictive of unfavorable outcome, such as mutations in isocitrate dehydrogenase 2 (IDH2-R172) and overexpression of the oncogenes brain and acute leukemia, cytoplasmic $(B A A L C)$ and v-ets erythroblastosis virus E26 oncogene homolog (ERG). In contrast, SPARC was downregulated in CN-AML patients harboring mutations in nucleophosmin (NPM1) that are associated with favorable prognosis. Based on these observations, we hypothesized that SPARC expression is clinically relevant in AML. Here, we found that SPARC overexpression is associated with adverse outcome in CN-AML patients and promotes aggressive leukemia growth in murine models of AML. In leukemia cells, SPARC expression was mediated by the SP1/NF- $\kappa$ B transactivation complex. Furthermore, secreted SPARC activated the integrinlinked kinase/AKT (ILK/AKT) pathway, likely via integrin interaction, and subsequent $\beta$-catenin signaling, which is involved in leukemia cell self-renewal. Pharmacologic inhibition of the SP1/NF- $\kappa$ B complex resulted in SPARC downregulation and leukemia growth inhibition. Together, our data indicate that evaluation of SPARC expression has prognosticative value and SPARC is a potential therapeutic target for AML.
\end{abstract}

\section{Introduction}

The secreted protein, acidic, cysteine-rich (SPARC) gene, located at chromosome bands 5q31-q33 (1), is highly conserved in vertebrates and encodes a $32-\mathrm{kDa}$ matricellular protein also known as osteonectin or BM-40 $(2,3)$ that regulates cell-matrix interactions and tissue remodeling through the binding of collagen and other extracellular matrix proteins and activation of matrix metalloproteinases (2). SPARC also interacts with and regulates several growth factors, including TGF- $\beta$, FGF, VEGF, and PDGF (4-7). This pleiotropic activity suggests that the SPARC protein is likely to be expressed when tissue undergoes normal or pathologic remodeling. SPARC expression has been shown to be altered in a variety of human conditions (e.g., diabetes, obesity) and in several types of cancer. However, the biologic and clinical significance of this gene is controversial, and conflicting reports have classified SPARC as either a tumor suppressor gene or an oncogene. The difficulty in assigning a specific function to the SPARC protein is related to the diverse roles that it can play both intracellularly in malig-

Authorship note: Clara D. Bloomfield and Guido Marcucci are co-senior authors and contributed equally to this work.

Conflict of interest: The authors have declared that no conflict of interest exists. Citation for this article: J Clin Invest. 2014;124(4):1512-1524. doi:10.1172/JCI70921. nant cells and extracellularly in the surrounding microenvironment (8). Low expression levels of SPARC were found in ovarian (9), colorectal $(10,11)$, and pancreatic cancer (12), whereas high SPARC expression was reported in breast cancer $(13,14)$, melanoma $(15,16)$, and glioblastoma (17). Stromal SPARC expression was associated with poor prognosis in non-small cell lung cancer (18) and with disease recurrence in breast ductal carcinoma in situ (19), whereas low stromal expression of SPARC predicted poor prognosis in colon cancer (20).

In hematologic malignancies, the role of SPARC is equally controversial. It was found to be downregulated at diagnosis in patients with del(5q) myelodysplastic syndromes (MDS) and upregulated following treatment with lenalidomide (21-23). SPARC was also found to be downregulated in acute myeloid leukemia (AML) with $M L L$ rearrangements, usually associated with unfavorable prognosis, and upregulated in AML with $t(8 ; 21)$ or inv (16), which is usually associated with favorable prognosis, although no correlation of SPARC expression with outcome was reported (24). In chronic myelogenous leukemia, the accumulation of intracellular SPARC mediated by the Fyn/ERK signaling pathway seemingly contributed to imatinib resistance (25).

Recently, we observed that SPARC was upregulated in gene expression profiles (GEPs) associated with prognostically unfa- 
A

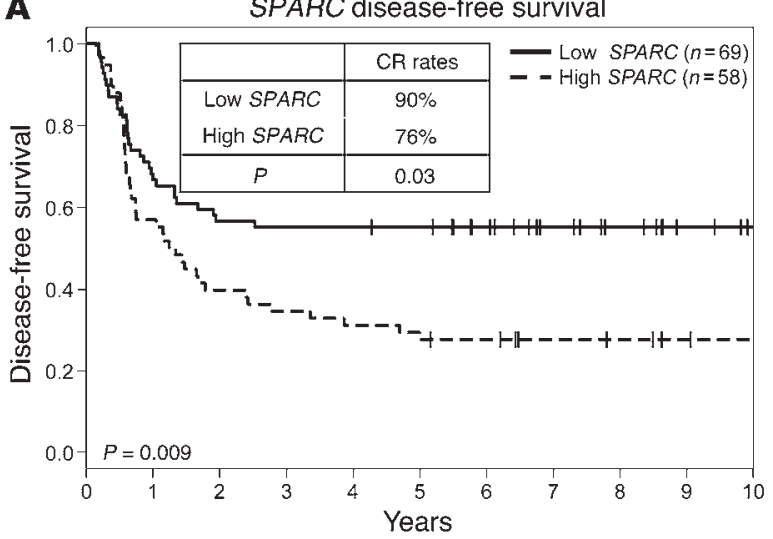

B

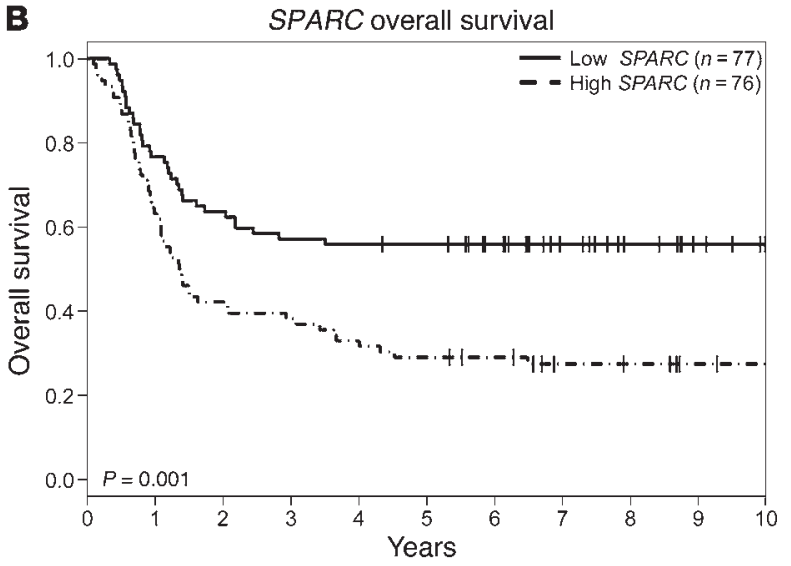

vorable gene mutations (i.e., those in isocitrate dehydrogenase 2 [IDH2-R172]; ref. 26) or overexpressed genes (i.e., brain and acute leukemia, cytoplasmic $[B A A L C]$ and v-ets erythroblastosis virus E26 oncogene homolog [ERG]; ref. 27), and downregulated in GEP associated with the prognostically favorable nucleophosmin (NPM1) mutation (28) in cytogenetically normal AML (CN-AML). This led us to hypothesize that SPARC overexpression contributes to a more aggressive phenotype in AML. Thus, we dissected the clinical significance of SPARC overexpression in AML, the mechanisms by which this gene is deregulated, and the downstream effects of this aberrantly expressed gene. We show that SPARC overexpression independently predicts adverse outcome in $\mathrm{CN}$ AML patients, thus representing what we believe to be a novel prognostic marker in AML. Consistent with these findings, we also demonstrate that SPARC contributes to leukemia growth in vitro and aggressive disease in vivo. SPARC overexpression activates integrin-linked kinase/AKT (ILK/AKT) and in turn $\beta$-catenin and could be targeted by modulating the SP1/NF-אB/miR-29b network, thereby also representing a potential therapeutic target in AML.

\section{Results}

SPARC overexpression is associated with adverse clinical outcome in $C N-A M L$. SPARC expression was analyzed by nCounter assays (NanoString Technologies) (29) in 153 younger (age range, 18-59 years) adults with primary CN-AML treated with cytarabinedaunorubicin-based regimens; clinical and molecular characteristics are shown in Supplemental Table 1 (supplemental material available online with this article; doi:10.1172/JCI70921DS1).

\section{Figure 1}

$S P A R C$ overexpression is associated with adverse clinical outcome in CN-AML. Outcome of CN-AML patients $(n=153)$ according to SPARC expression levels. Median value of the measured SPARC expression levels was used to dichotomize patients into high and low expressers. (A) CR rates and DFS. (B) OS.

Patients were dichotomized into higher and lower SPARC expressers using the median value cut-off. With a median follow-up of 8.7 years, higher SPARC expressers had lower odds of achieving a complete remission $(\mathrm{CR})(P=0.03)$ and shorter disease-free survival (DFS) $(P=0.009 ; 5$-year DFS $28 \%$ vs. $55 \%)$ and overall survival (OS) $(P=0.001 ; 5$-year OS $29 \%$ vs. $56 \%)$ than lower expressers (Figure 1, $\mathrm{A}$ and $\mathrm{B})$. In multivariable analyses, higher SPARC expression was independently associated with lower odds of CR $(P=0.007)$, once adjusting for white blood count (WBC) $(P=0.003)$, and shorter OS $(P=0.03)$, once adjusting for FLT3 internal tandem duplication (FLT3-ITD) $(P<0.001)$, WT1 $(P=0.003)$, and RUNX1 $(P=0.006)$ mutations and $\mathrm{WBC}(P<0.001)$. There was also a trend for shorter DFS $(P=0.08)$ once adjusting for FLT3-ITD $(P<0.001)$ and WT1 mutation $(P=0.004)$. These data support the notion that SPARC is differentially expressed across AML patients and that the difference in the SPARC expression in this patient population has potentially biologic and clinical relevance.

SPARC promotes leukemia growth in vitro and an aggressive disease in vivo. Having shown that higher SPARC expression was associated with adverse outcome, next we interrogated the SPARC-dependent mechanisms mediating the aggressive phenotype. THP-1 cells express a relatively lower endogenous level of SPARC mRNA (Supplemental Figure 1) and therefore represent a suitable in vitro model for gain-of-function experiments. THP-1 cells were stably infected with pLenti-SPARC (THP-1/SPARC) or empty vector as a control (THP-1/EV). Ectopic expression of SPARC was confirmed by Western blotting (Figure 2A). A significant increase in the ability of colony formation was observed in THP-1/SPARC cells compared with the THP-1/EV cells (mean, 209 vs. $72 ; P=0.007$; Figure 2A). Likewise, when we knocked down SPARC (Figure 2B) in Kasumi-1 cells that express a relatively higher endogenous levels of the gene (Supplemental Figure 1), we observed a decrease in the colony-forming ability in SPARC siRNA-transfected cells compared with the control siRNA-transfected cells (mean, 106 vs. 50; $P=0.002$; Figure 2B). Similar experiments were also conducted in primary blasts from 5 AML patients (numbers 1-5) expressing a relatively lower level of endogenous SPARC and 2 patients (numbers 6, 7) expressing a relatively higher level of endogenous SPARC (see patients' cytogenetic and molecular features in Supplemental Table 2). For patients 1-5; blasts were infected with either the pLenti-SPARC or the pLenti-EV vector. A significant increase in colony forming ability was observed in SPARC-infected blasts compared with EV controls for patients $1-4$ (means, 16 vs. 7; $P=0.005 ; 109$ vs. $73 ; P=0.02 ; 23$ vs. $16 ; P=0.006$ and 357 vs. 170 ; $P=0.06$ ) (Figure 2C). In blasts from a patient 5 , ectopic SPARC expression did not significantly increase the colony-forming ability after first plating, but a significant increase in the number of colonies was observed after the secondary plating (mean, 80 vs. 3; $P=0.001$; Figure 2D). For patients 6 and 7 , we observed an increase in spontaneous apoptosis in AML blasts when SPARC endogenous expression was knocked down by siRNAs (Figure 2E), suggesting that SPARC promotes both growth and survival of leukemia cells. 

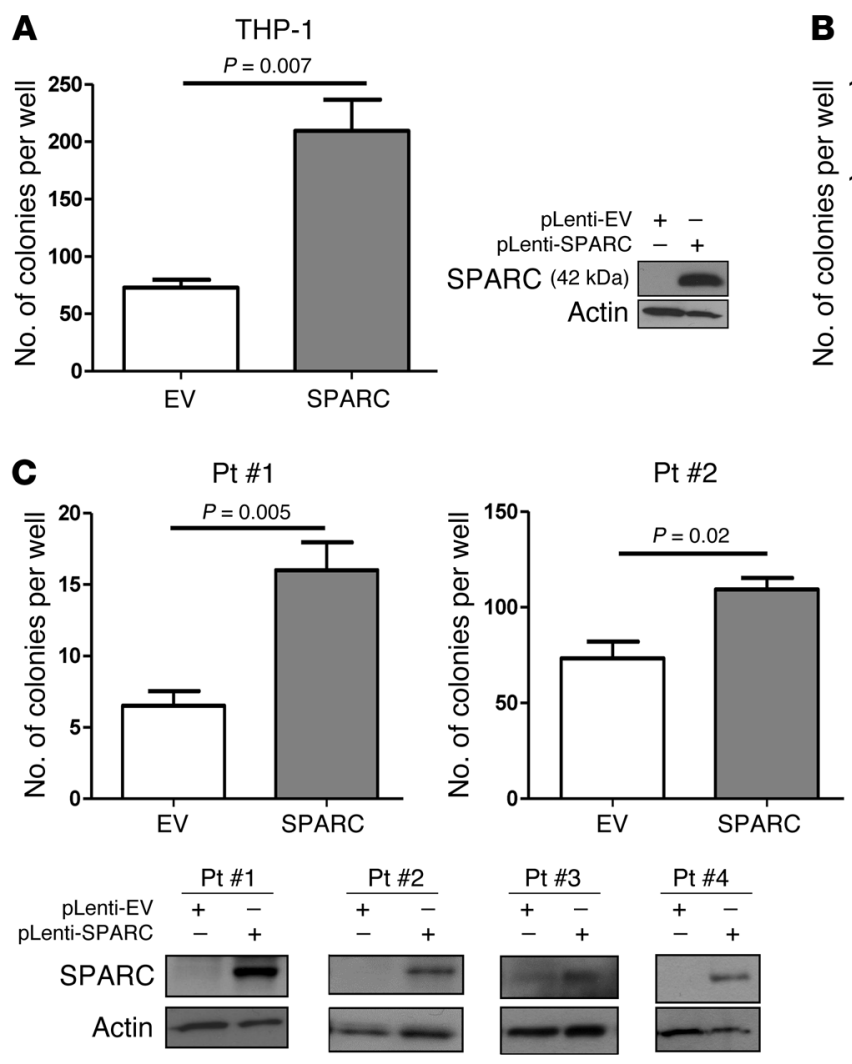

kDa

B

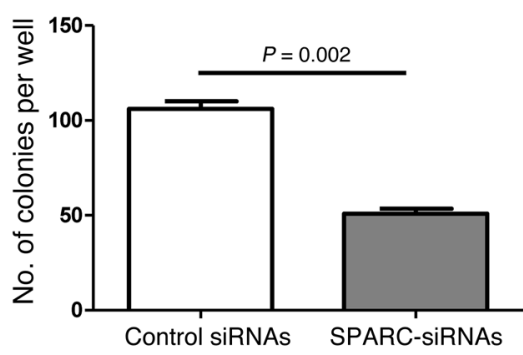

Pt \#3
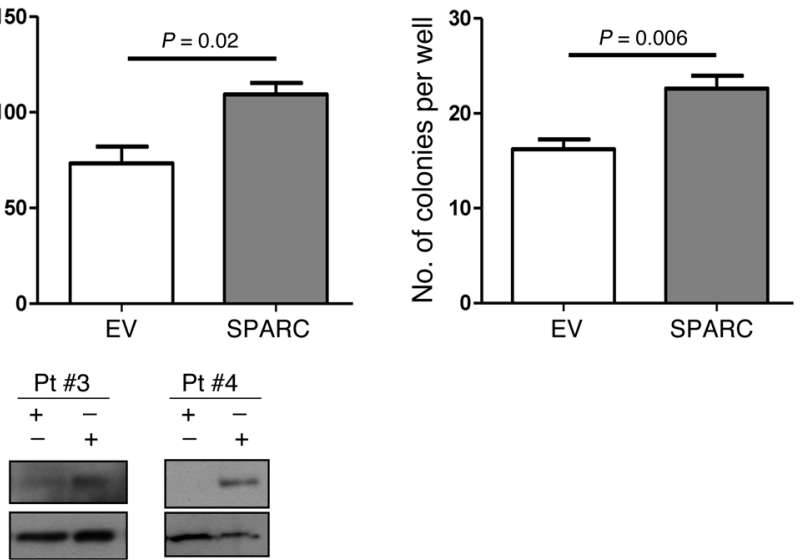

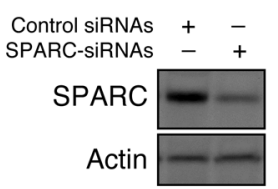

Actin
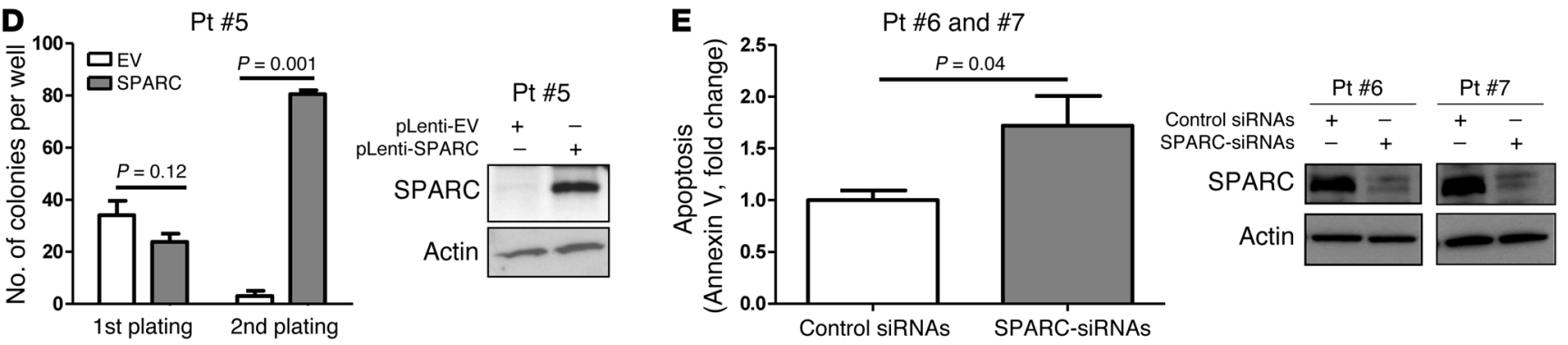

Figure 2

SPARC promotes growth advantage in AML. (A) SPARC protein levels (right panel) and quantification of colony formation (left panel) in THP-1 cells ectopically expressing SPARC. (B) SPARC knockdown (right panel) and quantification of colony formation (left panel) in Kasumi-1 cells. (C) SPARC ectopic expression in primary blasts from 4 AML patients (numbers 1-4; bottom panel) and quantification of colony formation assays in these cells (top panel). (D) Western blotting showing SPARC protein levels (right panel) and quantification of colony formation in primary blasts from AML patient 5 ectopically expressing SPARC scored at 14 days following first and second plating (left panel). (E) SPARC protein levels measured in primary blasts from 2 AML patients (numbers 6-7) transfected with SPARC siRNA (right panel) and quantification of apoptosis determined by annexin V staining in SPARC-siRNAs versus control siRNA-transfected blasts (left panel). Data represent mean \pm SEM.

To examine the contribution of SPARC to leukemia growth in vivo, NOD/SCID/gamma (NGS) mice were injected with THP-1/ SPARC cells or THP-1/EV cells via tail vein. Eight weeks later, 3 mice in each group were sacrificed. Bone marrow engraftment of the leukemia cells was confirmed by CD45 antibody staining. The THP-1/SPARC mice had significantly larger livers $(P=0.04$; Figure $3 \mathrm{~A})$ and spleens $(P=0.008$; Figure $3 \mathrm{~B})$ than THP-1/EV controls. THP-1/SPARC cells formed coalescing masses obliterating large expanses of the liver parenchyma (Supplemental Figure 2), while THP-1/EV cells were observed either alone or in small clusters within hepatic sinusoids without effacing the hepatic cords.
The THP-1/SPARC cells had a monomorphic phenotype and a seemingly higher proliferative capacity compared with the more pleomorphic character and increased apoptotic tendency of the THP-1/EV cells (see immunohistochemistry (IHC) staining for SPARC, CD45, and Ki67 in Figure 3C and quantification of Ki67 in Figure 3D). Histopathology of spleen sections showed increased infiltration of leukemic cells and destruction of splenic architecture in tissues obtained from THP-1/SPARC mice compared with tissues obtained from THP-1/EV mice (Supplemental Figure 2). The THP-1/SPARC mice $(n=9)$ also had a significantly shorter survival than the THP-1/EV mice $(n=9)\left(2 \times 10^{6}\right.$ cells were injected 
A THP-1/EV THP-1/SPARC

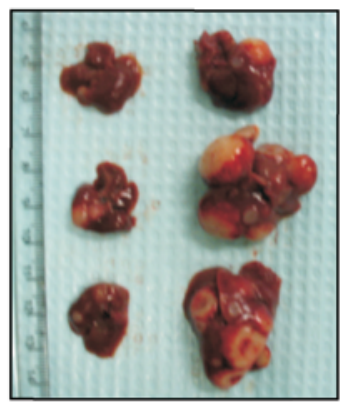

B

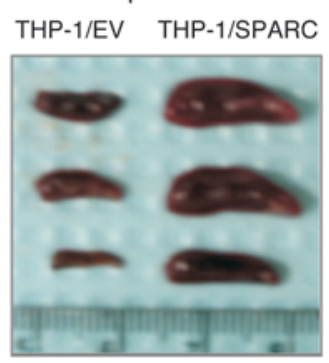

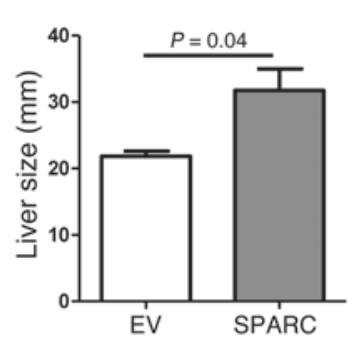

C THP-1/EV

के THP-1/EV

THP-1/SPARC

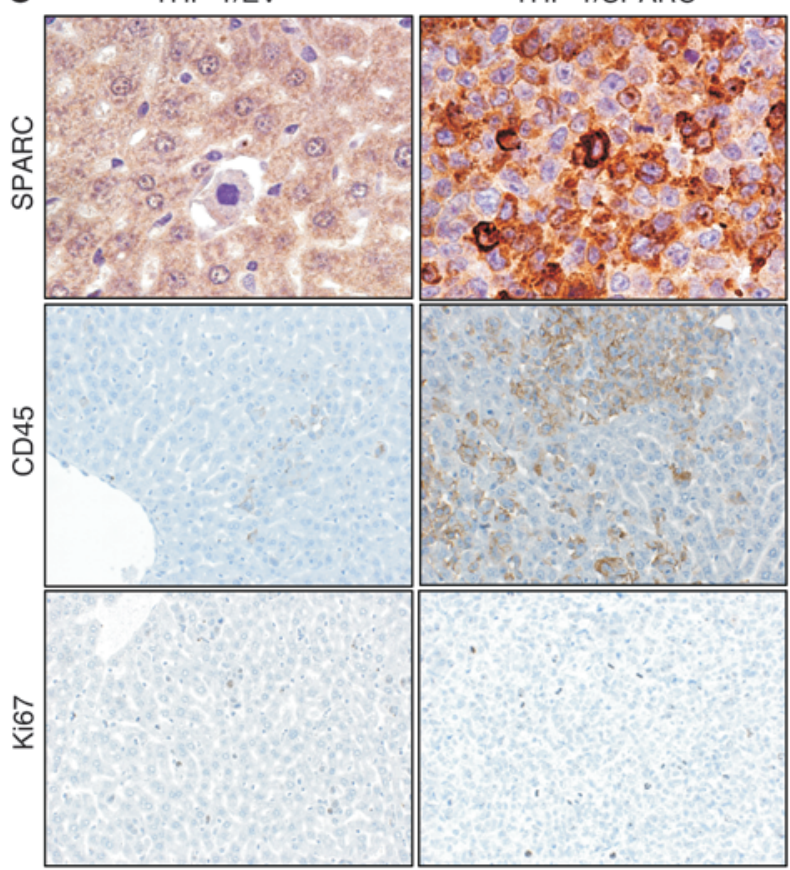

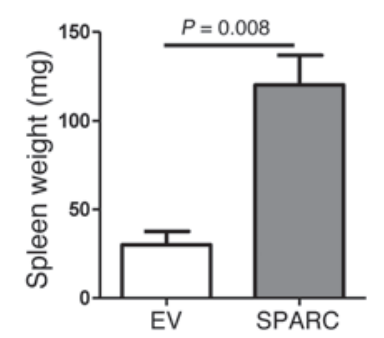

D

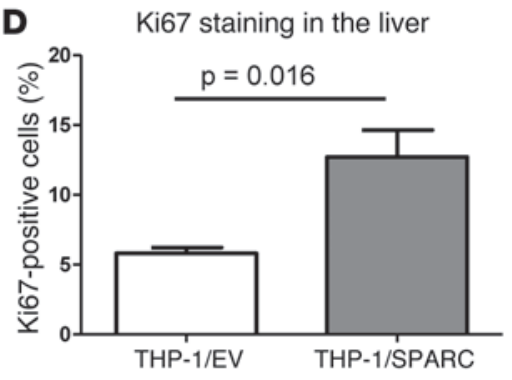

E

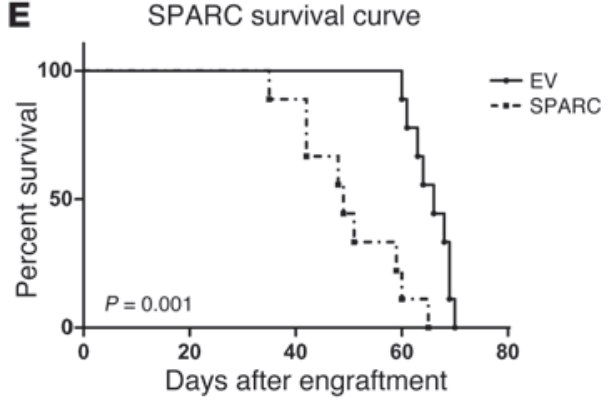

Figure 3

SPARC promotes aggressive disease in vivo. NSG mice were engrafted with THP-1 cells infected with pLenti-EV or pLenti-SPARC vectors; mice were sacrificed and tissues were collected 8 weeks following engraftment. (A) Liver sizes (mean \pm SEM). (B) Spleen sizes (mean \pm SEM). (C) Immunohistochemical staining for SPARC, CD45, and Ki67 expression in liver tissues obtained from THP-1/EV and THP-1/SPARC mice. Original magnification, $\times 400$. (D) Quantitative analysis for Ki67 (proliferation marker) expression in liver tissues obtained from THP-1/EV and THP-1/SPARC mice (using ImmunoRatio Software). (E) Survival analysis of THP-1/SPARC mice $(n=9)$ compared with THP-1/EV controls $(n=9)$.

per mouse; median survival, 49 vs. 66 days; $P=0.001$; Figure 3E). Similar results were obtained in a repeated experiment where a higher number of cells $\left(5 \times 10^{6}\right)$ were injected into each mouse (Supplemental Figure 3). Consistent with these results, mice injected with the SPARC-shRNA-infected CG-SH cells, which otherwise express high levels of endogenous SPARC, showed a trend for smaller spleens than mice injected with control shRNA-infected cells (Supplemental Figure 4).

SPARC activates ILK/AKT/B-catenin signaling pathways. Next, we asked how SPARC contributes to the mechanisms of leukemia growth. Several cell-surface receptors have been identified as interacting with the secreted SPARC protein, including some of the membrane integrins $(30,31)$. This interaction activates ILK, a multifunctional cytoplasmic serine/threonine kinase (32-34) that phosphorylates and activates AKT and phosphorylates and inactivates glycogen synthase kinase $3 \beta$ (GSK3 $\beta$; Ser9). GSK3 $\beta$ promotes $\beta$-catenin degradation, but once phosphorylated, its activity is suppressed, and $\beta$-catenin stabilizes and translocates into the nucleus (35). Activated AKT phosphorylates and stabilizes $\beta$-catenin at residue Ser552 (36). Thus, the net result of the SPARC/integrin/ILK interplay is enhanced $\beta$-catenin. The latter is required for self-renewal of leukemia stem cells (LSCs) (37-41). Thus, we hypothesized that SPARC overexpression leads to aberrant $\beta$-catenin activation in AML.

SPARC is a secreted protein $(2,3)$. We found significantly higher levels of SPARC in the media collected from THP-1/SPARC cell cultures than in the media collected from THP-1/EV cell cultures (Figure 4A). We incubated THP-1 cells with conditioned media from THP-1/SPARC cells and THP-1/EV cells and performed colony assay 48 hours after incubation. We observed higher num- 
A

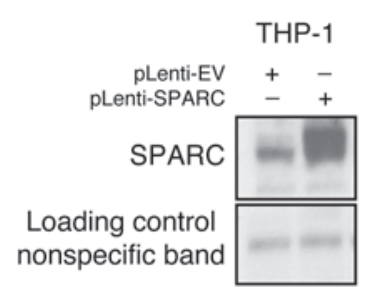

\section{B}
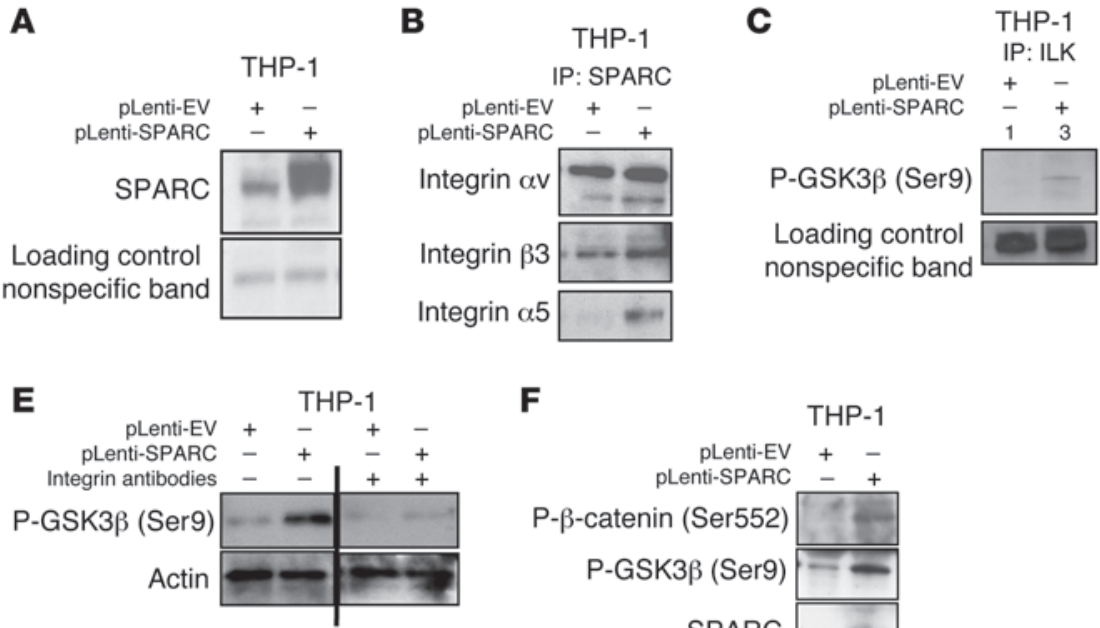

$\mathbf{F}$

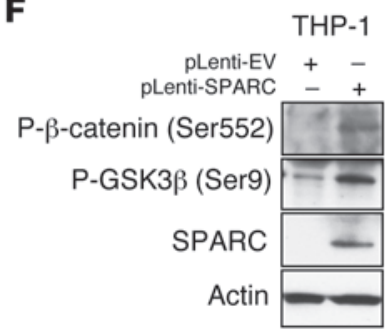

D

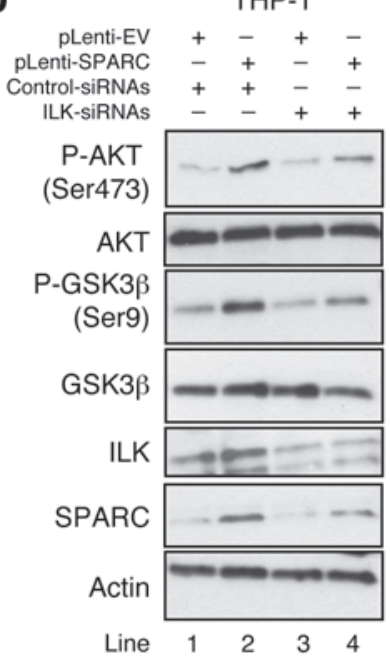

H

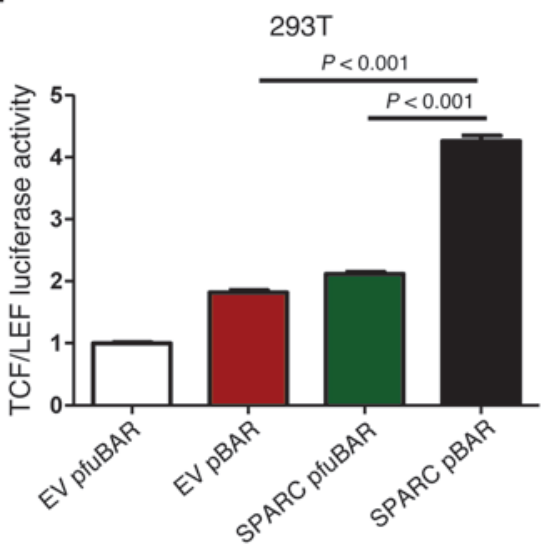

G
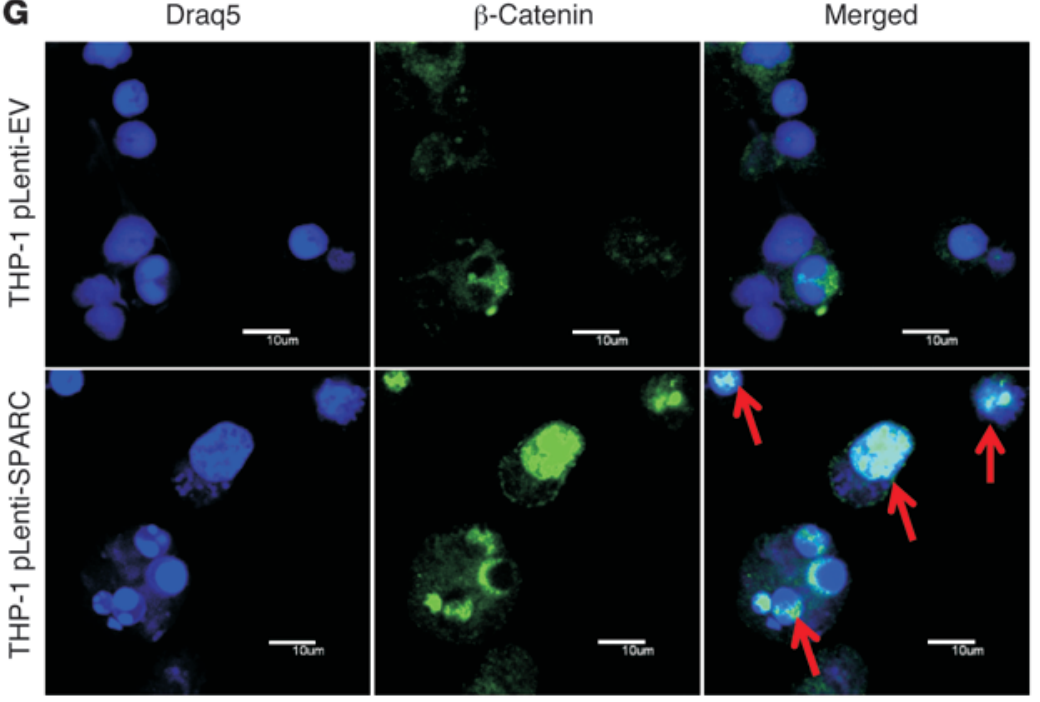

$\mathbf{J}$

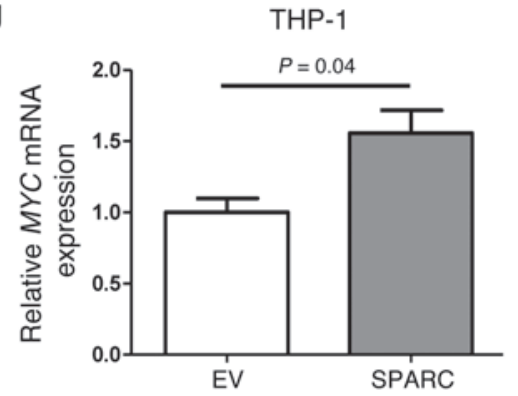

THP-1

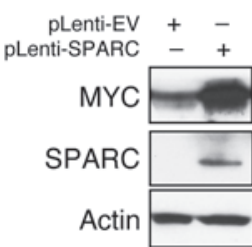




\section{Figure 4}

SPARC activates ILK/AKT/ $\beta$-catenin signaling pathways in THP-1 cells and enhances $\beta$-catenin nuclear translocation and transcriptional activity. THP-1 cells were transfected with pLenti-EV or pLentiSPARC vectors. (A) Western blot measuring secreted SPARC protein levels in media collected from cell culture of THP-1 cells infected with pLenti-SPARC vector compared with cells infected with pLenti-EV. (B) SPARC and integrin coimmunoprecipitation assay. (C) ILK kinase activity assay. Lysates from THP-1/SPARC cells and THP-1/EV cells were immunoprecipitated with anti-ILK antibody and assayed utilizing GSK3 fusion protein (ILK substrate). Numbers represent ratio of band intensity to control band. (D) Western blot analysis of P-AKT(Ser473) and P-GSK3 $\beta$ (Ser9) levels in THP-1/SPARC cells compared with THP-1/ $\mathrm{EV}$ in the presence of endogenous ILK or following ILK knockdown or (E) following blocking integrin receptors with integrin antibodies. Lanes separated by a line were run on the same gel but were noncontiguous. (F) Western blot analysis of P- $\beta$-catenin (Ser552) in THP-1/SPARC cells compared with THP-1/EV cells. (G) Immunofluorescence confocal microscopy images showing $\beta$-catenin (green) and cell nuclei (blue) in EV- or SPARC-transfected THP- 1 cells stained with $\beta$-catenin antibody and Draq5 antibody (nuclear staining); arrows indicate cells exhibiting $\beta$-catenin nuclear translocation. Scale bars: $10 \mu \mathrm{m}$. (H) Luciferase activity measured in 293T cells following cotransfection with pBAR (TCF/ LEF reporter vector) or pfuBAR (TCF/LEF reporter vector with mutated site) and pLenti-EV or pLenti-SPARC and (I) in the presence of control siRNAs or ILK siRNAs. (J) MYC mRNA expression (left panel) and protein expression (right panel) in THP-1/SPARC cells compared with THP-1/EV. Data represent mean \pm SEM for 3 different experiments.

ber of colonies in THP-1/SPARC media incubated THP-1 cells compared with THP-1/EV media incubated cells (Supplemental Figure 5), suggesting that the conditioned media from SPARC transfected cells induce cell growth in SPARC-nonexpressing cells. Coimmunoprecipitation assays with SPARC and surface integrins showed binding of the protein at least to integrins $\alpha_{v}, \beta_{3}$, and $\alpha_{5}$ (Figure 4B) and lower or no binding to integrins $\beta_{1}, \alpha_{4}$, and $\beta_{4}$ (data not shown). Kinase activity assay of anti-ILK immunoprecipitate from THP-1/SPARC cells incubated with recombinant GSK3 showed increased P-GSK3 $\beta$ (Ser9) (mean $2.4 \pm 0.68$ of 3 different experiments) as compared with the immunoprecipitate from THP-1/EV cells, thereby reflecting higher ILK activity in the presence of SPARC (Figure 4C). Furthermore, increased endogenous P-AKT(Ser473) and P-GSK3 $\beta$ (Ser9) were detected in THP-1/ SPARC cells compared with THP-1/EV cells (Figure 4D, lines 1 and 2). Knocking down ILK (Figure 4D, lines 3 and 4) decreased AKT and GSK3 $\beta$ phosphorylation in THP-1/SPARC cells. Consistently, functional inhibition of ILK activity using the T315 (ILK inhibitor) (42) abolished SPARC-induced cell growth as assessed by MTS and colony-forming assay (Supplemental Figure 6).

ILK activates AKT through PI3K activation (43), suggesting that pharmacologic blockade of PI3K signaling should result in abrogating the SPARC effect on AKT and GSK3 $\beta$ phosphorylation. Indeed, THP-1/SPARC cells treated with 50 nM PI3K inhibitor LY294002 showed decreased AKT(Ser473) and P-GSK3 $\beta$ (Ser9) compared with untreated THP-1/SPARC cells (Supplemental Figure 7).

Next, we tested whether blocking integrin receptors would decrease SPARC function. GSK3 $\beta$ phosphorylation in THP-1/ SPARC cells was reduced in THP-1/SPARC cells incubated with antibodies against $\alpha_{v}, \beta_{3}$, and $\beta_{1}$ (Figure $4 \mathrm{E}$ ); indeed, blocking $\alpha_{v} \beta_{3}$ was sufficient to decrease SPARC-dependent GSK3 $\beta$ phosphorylation (Supplemental Figure 8). Consistent with these findings, we observed higher levels of $\mathrm{P}-\boldsymbol{\beta}$-catenin(Ser552) (Figure $4 \mathrm{~F}$ ) and an increased $\beta$-catenin nuclear translocation (Figure 4G) in THP-1/ SPARC compared with THP-1/EV cells.

Once in the nucleus, $\beta$-catenin interacts with TCF/LEF transcription factors and promotes expression of genes supporting cell growth and proliferation (e.g., MYC and CCND1). Ectopic SPARC expression resulted in an approximately 4-fold increase in TCF/LEF reporter activity, as measured by luciferase assay in SPARC-transfected 293T cells compared with EV-transfected controls $(P<0.001$; Figure $4 \mathrm{H})$. The SPARC-induced TCF/LEF activity decreased upon ILK siRNA knockdown (Figure 4I). SPARC protein levels in $293 \mathrm{~T}$ cells assessed by Western blot are shown in Supplemental Figure 9. Increase in SPARC-induced $\beta$-catenin-TCF/LEF activity was also supported by upregulation of the TCF/LEF target gene MYC (Figure $4 \mathrm{~J}$ ). We also observed consistent results in other cell lines (e.g., MV4-11 cells) forced to express SPARC (Supplemental Figure 10).

Finally, we validated our findings in blasts from AML patients. Forced expression of SPARC increased P- $\beta$-catenin (Ser552) (Figure 5A; patient 2), while siRNA SPARC knockdown decreased $\mathrm{P}-\beta$-catenin(Ser552) (Figure 5B; patient 6 ) and total $\beta$-catenin (Figure 5C; patient 8). SPARC overexpression and knockdown in primary blasts resulted, respectively, in significant increase and decrease of MYC mRNA expression (Figure 5, D and E). To ensure that increased TCF/LEF target gene expression was not due to other leukemogenic mechanisms operative in AML cells, we also showed that forced SPARC could increase MYC and CCND1 expression in $\mathrm{CD} 34^{+}$cells from cord blood cells of normal donors (Supplemental Figure 11).

It has been reported that $\beta$-catenin is expressed and activated in LSCs and that it is required for self-renewal $(37,38)$. Thus, we postulated that SPARC expression may be higher in less differentiated hematopoietic cell subpopulations. To prove this, we showed that SPARC expression was significantly higher in the CD34 ${ }^{+}$ compared with CD34- cells in normal bone marrow cells (NBM) $(n=4 ; P<0.001$; Figure $6 \mathrm{~A})$, and in $\mathrm{CD}^{+} 4^{+} / \mathrm{CD} 38^{-} \mathrm{HSCs} / \mathrm{multipo-}$ tent progenitors (HSC/MPP) compared with $\mathrm{CD} 34^{+} / \mathrm{CD} 38^{+}$cells (common-myeloid progenitors [CMP] and granulocyte-monocyte progenitors [GMP]) both in NBM $(n=3)$ and AML blasts $(n=3$; patients n. 1,5 and 9) (Figure 6, B and C). Furthermore, we showed that SPARC-transduced primary AML blasts (patient 9) indeed exhibited a higher percentage of engraftment in NSG mice than those transduced with GFP/EV control (3.1\% vs $0.4 \%$ circulating blasts 12 weeks after blast i.v. injection; Figure 6D).

$S P 1, N F-\kappa B$, and miR-29b modulate SPARC expression in AML. Next, we asked which mechanisms lead to aberrant SPARC expression in AML. We identified binding sites for SP1, NF-кB, and RUNX1 in the $(-651 / 1)$ promoter region (Transcriptional Regulatory Element Database [TRED] website; http://rulai.cshl.edu/cgi-bin/ TRED/tred.cgi?process=home) and binding sites for $m i R-29$ in the 3' UTR region of the human SPARC gene. Indeed, SPARC has been reported to be a miR-29 target (44). Notably, we have recently shown that activation and overexpression of oncogenes involved in AML (i.e., KIT, FLT3, and DNMTS) require interaction and transactivation of the transcription factors SP1 and NF-KB and inhibition of $m i R-29 b$ (45). Therefore, we postulated that a similar mechanism could be responsible for SPARC overexpression.

To test this hypothesis, we first performed luciferase gene reporter assays by cloning the SPARC promoter region spanning the SP1-, NF-кB-, and RUNX1-binding sites into the pGL4.11 luciferase vector. Then, $293 \mathrm{~T}$ cells were cotransfected with the reporter gene and vectors expressing SP1 and p65 (NF-кB subunit). A signifi- 
A

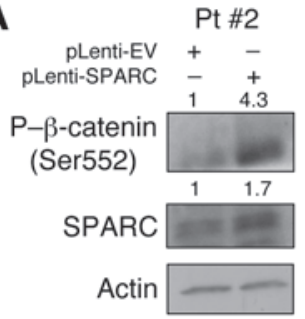

C
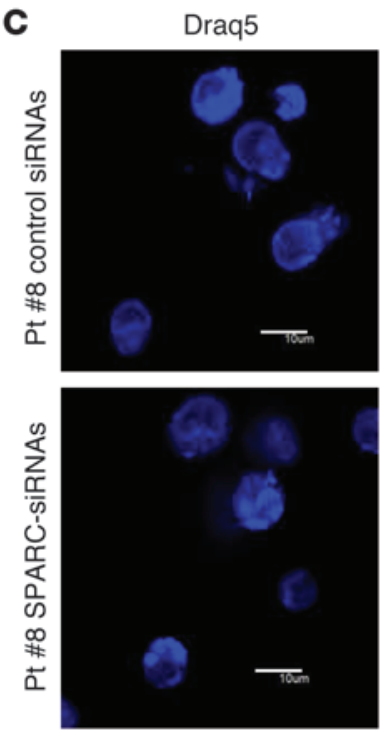

\section{D}

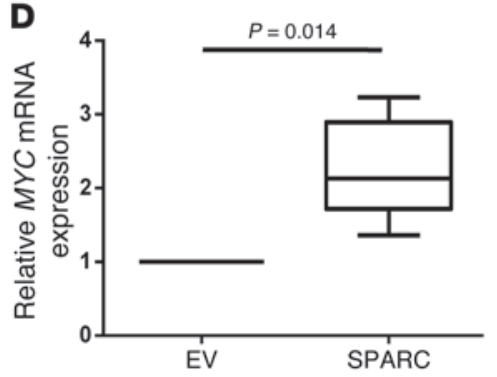

B
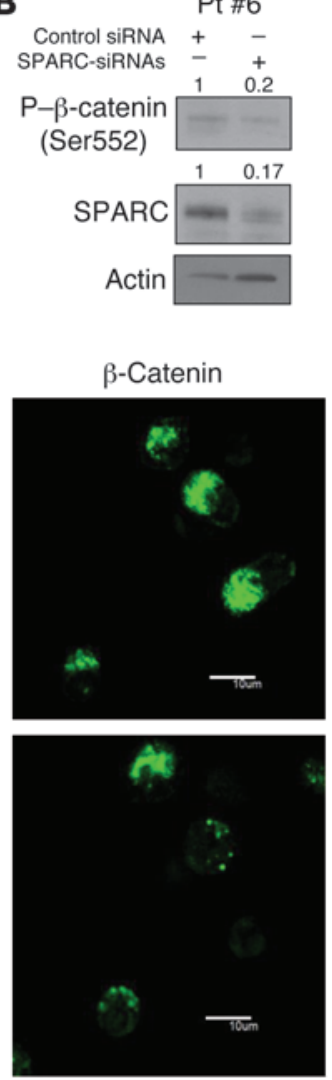
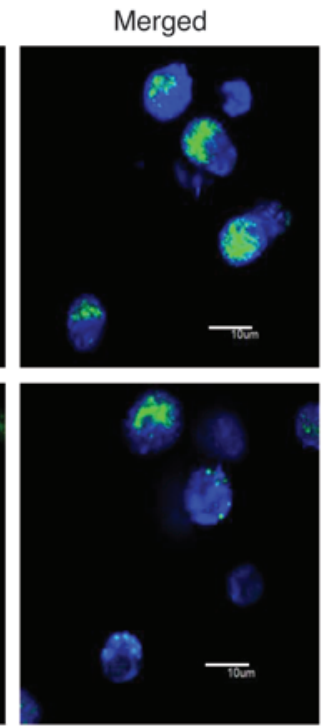

E

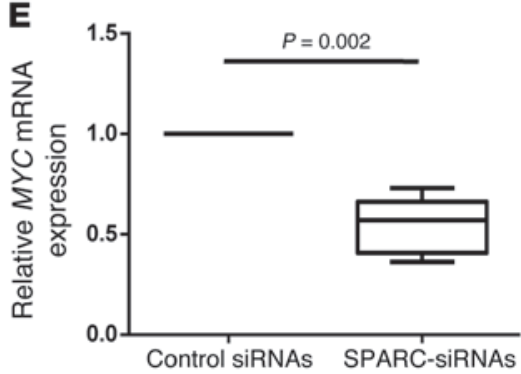

\section{Figure 5}

SPARC activates ILK/AKT/ $\beta$-catenin signaling pathways in primary blasts. (A) $\mathrm{P}-\beta$-catenin (Ser552) in primary blasts (AML patient 2 ) ectopically expressing SPARC and (B) in primary blasts (AML patient 6) transfected with SPARC siRNAs. Numbers represent ratio of band intensity to the control band. (C) Immunofluorescence confocal microscopy images showing $\beta$-catenin (green) and cell nuclei (blue), respectively, in primary blasts (AML patient 8 ) stained with $\beta$-catenin antibody and Draq5 antibody (nuclear staining) following SPARC knockdown. Scale bars: $10 \mu \mathrm{m}$. (D) Box plots showing MYC mRNA expression measured in primary blasts transfected with pLenti-SPARC compared with those transfected with pLenti-EV $(n=3)$. (E) Box plots showing MYC mRNA expression measured in primary blasts transfected with SPARC siRNAs compared with those transfected with control-siRNAs $(n=4)$. The boxes correspond to the 25th and 75th percentiles, the whiskers correspond to the minimum to maximum values, and the horizontal lines indicate the median. cant increase in luciferase activity was found in cells transfected with SP1- or $p 65$-expressing vectors compared with EV controls $(P<0.001$ for both; Figure 7A). Ectopic expression of SP1 and p65 also resulted in significant increases in endogenous SPARC expression in THP-1 cells (Figure 7, B and C), whereas SP1 and p65 siRNA knockdown resulted in significant decrease of SPARC expression in Kasumi-1 cells (Figure 7, D and E).

A significant decrease of SPARC promoter luciferase activity was found in 293T cells transfected with RUNX1 expression vector $(P<0.001 ;$ Figure 7F). In contrast, RUNX1-WT knockdown resulted in SPARC upregulation in MV4-11 cells (Figure 7G), while knockdown of RUNX1-RUNX1T1 translocation by siRNAs resulted in a significant SPARC downregulation in RUNX1-RUNX1T1-positive Kasumi-1 cells (Figure 7H).

Notably, ectopic expression of p65 and SP1 increased their respective enrichment on the SPARC promoter (Figure 7, I and J), and forced RUNX1-WT expression decreased SP1, but not p65, enrichment as measured by ChIP in THP-1 cells (Figure 7K).
These results are also consistent with the finding that RUNX1mutated CN-AML patients exhibit higher SPARC expression when compared with the RUNX1-WT patients (Supplemental Figure 12). Together, these results indicate that SP1, p65 and RUNX1 loss-of function promoted SPARC expression, while RUNX1-WT inhibited SPARC expression.

As SPARC was found to be a target for $m i R-29 a$ and $m i R-29 c$ (44) and the SPARC 3' UTR region was predicted to have binding sites for $m i R-29 b(46,47)$, we cloned the SPARC 3' UTR region in luciferase reporter vector ( $\mathrm{pGL} 3$ ) and cotransfected this construct with the synthetic microRNA ( $m i R-29 b$ and $m i R-9 *$ ) or scramble oligos in $293 \mathrm{~T}$ cells. We found a $60 \%$ decrease of SPARC 3' UTR-luciferase activity in cells cotransfected with $m i R-29 b$ compared with the activity in cells cotransfected with scramble $(P<0.001)$ or an unrelated microRNA: $m i R-9 *$ (Figure 8A). Kasumi-1 cells transfected with $m i R-29 b$ showed significant decreases in endogenous SPARC (Figure 8B). AML blasts transfected with $m i R-29 b$ also showed significant decreases in SPARC levels at 24 hours (Figure 8C). 
A

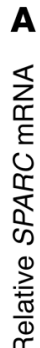

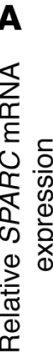

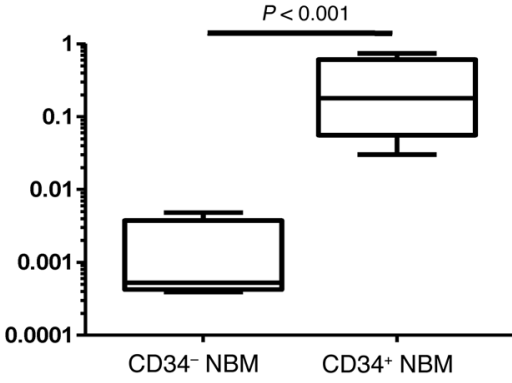

HSC/MPP: CD34 $4^{+} / \mathrm{CD} 38$

CMP: CD34 $/$ CD45RA $-/ C D 123^{10}$

GMP: $\mathrm{CD} 34^{+} / \mathrm{CD} 45 \mathrm{RA}^{+} / \mathrm{CD} 123^{\circ}$

B
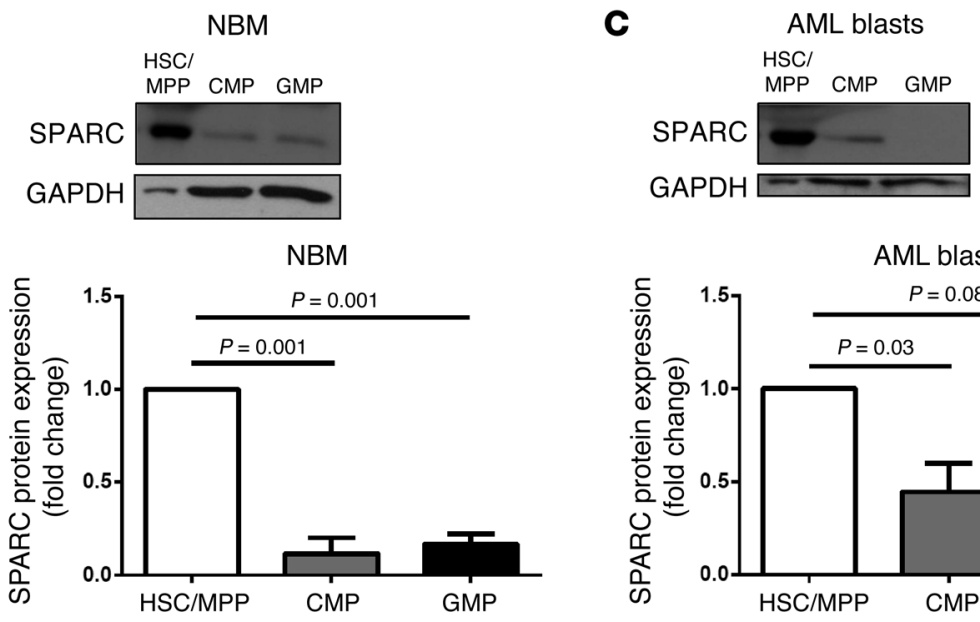

C

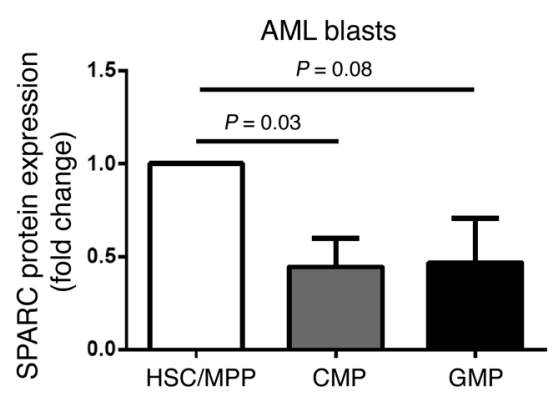

Figure 6

SPARC expression is higher in normal and AML immature cell subpopulations and enhances blast engraftment in immunodeficient mice. (A) Box plot showing SPARC mRNA level measured in CD34+ and CD $34^{-}$cells of NBM samples $(n=4)$. The boxes correspond to the 25th and 75th percentiles, the whiskers correspond to the minimum to maximum values, and the horizontal lines indicate the median. (B) SPARC protein expression measured by Western blot in sorted stem cell/progenitor populations of NBM samples $(n=3)$ and (C) AML blasts $(n=3)$; measurements are presented by mean \pm SEM. (D) Percentage of engraftment of primary AML blasts from AML patient 9 transduced with either MIGRSPARC or MIGR-EV in NSG mice $(n=2)$; measurements are presented by mean \pm SEM.
We have previously shown that $m i R-29 b$ is downregulated in AML via a SP1/NF-кB/HDAC inhibitory complex and that pharmacologic disruption of this complex results in miR-29b upregulation and downregulation of direct (i.e., SP1, DNMT3A, DNMT3B) and indirect (i.e., DNMT1, FLT3, KIT) miR-29b targets $(45,48,49)$. Consistent with these reports, we showed that bortezomib at concentrations achievable in vivo $(45,50)$ significantly increased $m i R$ $29 b$ expression (Figure 8D) and in turn decreased SP1 and SPARC mRNA and protein levels in Kasumi-1 cells (Figure 8, E and F) and in blasts from 3 different patients with primary AML (numbers 6 , 10, and 7) (Figure 8, G and H). Consistent with the in vitro results, we found SPARC expression was significantly reduced in blood and spleen from CG-SH cell-engrafted NSG mice treated with bortezomib $(1 \mathrm{mg} / \mathrm{kg})$ twice a week for 4 weeks compared with vehicle-treated mice (Supplemental Figure 13).

\section{Discussion}

Although altered SPARC expression has been observed in cancer, the clinical significance of both upregulation and downregulation of the gene and the encoded protein remain to be fully elucidated $(15,17,51-58)$. In contrast with solid tumors, the number of reports investigating this gene in hematologic malignancies is relatively small. In MDS, 5q deletion results in allelic loss and significant downregulation of SPARC expression in the progenitor compartment $(22,23)$, and a dramatic upregulation of SPARC occurs in response to lenalidomide (23). Although this suggests that SPARC expression is a treatment-response predictor and perhaps plays a role in normal hematopoiesis, to date, no biological evidence supporting this notion has been reported. Indeed, SPARC was found to be dispensable for murine hematopoiesis (59). Nev- ertheless, the complexity of the hematopoietic role of SPARC is illustrated by a recent report (60). These authors showed in a myeloproliferative murine model that increased SPARC expression in the bone marrow stroma favored fibrotic changes, while loss of SPARC expression resulted in a defective stromal niche (60).

Here, we sought to dissect the role of SPARC in AML, starting from the observation that this gene was found overexpressed in GEPs associated with distinct molecular subsets of patients harboring prognostically unfavorable mutations or overexpressed genes. Supporting the hypothesis that SPARC overexpression likely contributes to aggressive myeloid leukemogenesis, we showed that high SPARC expression was associated with adverse outcomes in CN-AML patients. Although SPARC overexpression was associated with other unfavorable molecular markers at diagnosis, in multivariable models for outcome, the impact of SPARC remained significant even after adjusting for other molecular prognosticators, supporting an independent role of this gene in determining an aggressive clinical phenotype. To our knowledge, this is the first study reporting the clinical relevance of SPARC expression in AML. We validated these findings through mechanistic experiments using AML preclinical models and primary blasts. We showed that SPARC upregulation promoted an aggressive phenotype in AML cells. Mice engrafted with AML cells forced to express SPARC had increased leukemia burden and shorter survival compared with controls.

The mechanisms leading to SPARC upregulation in AML blasts are multifactorial and likely converge to NF- $\mathrm{B}$-dependent pathways, which have been previously shown to be constitutively activated in AML LSCs (61). We found that SPARC may function through an autocrine mechanism; once secreted by AML cells, 

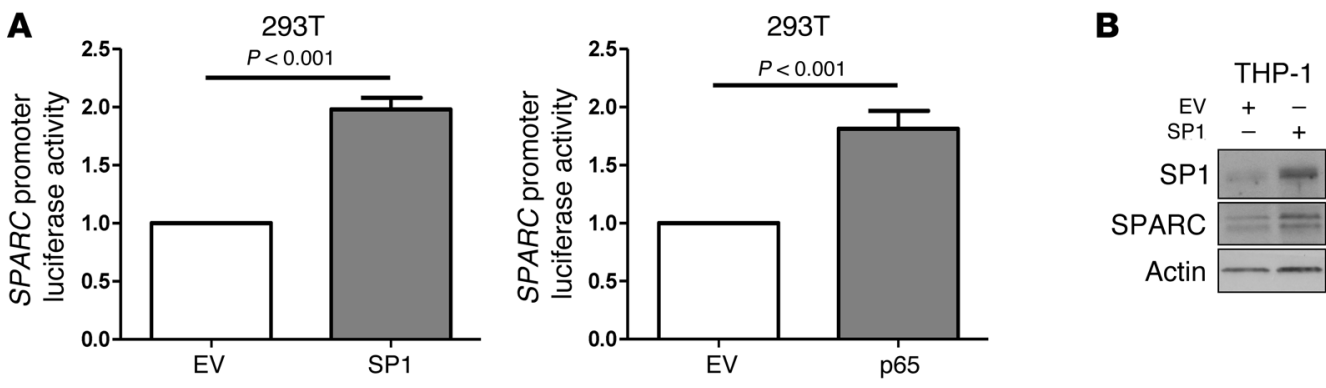

C

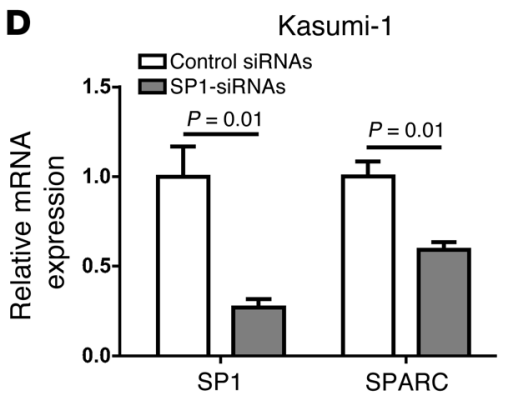

E

Kasumi-1

$\mathbf{F}$
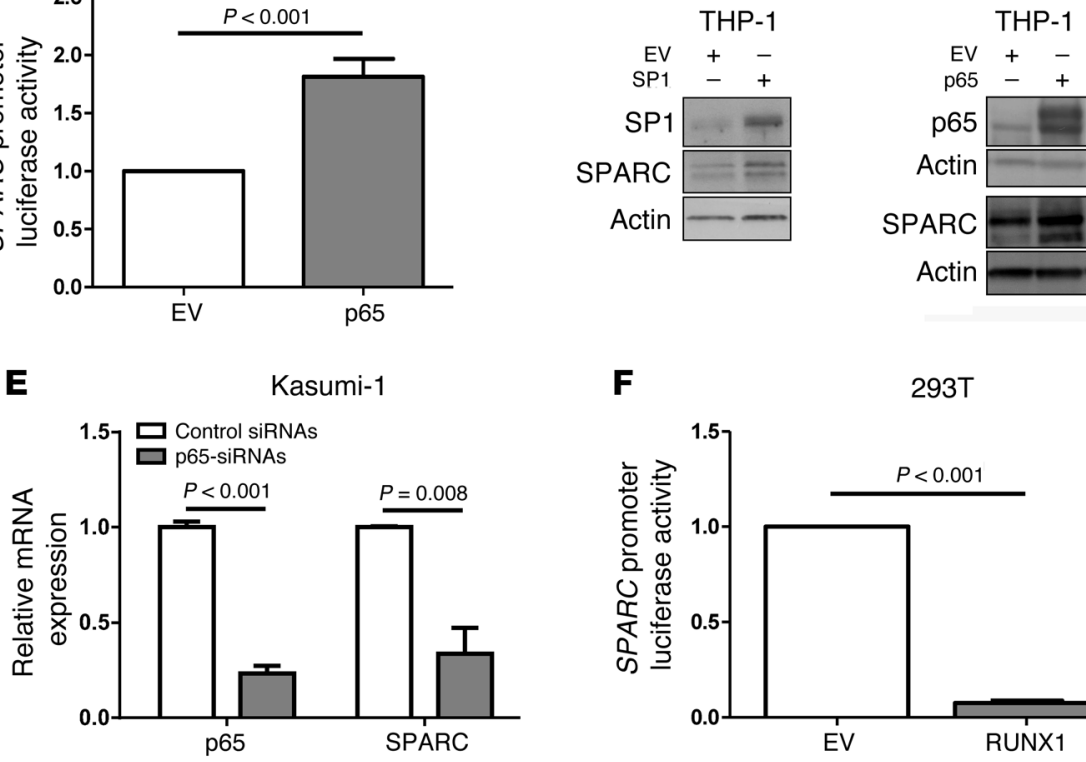

$293 T$
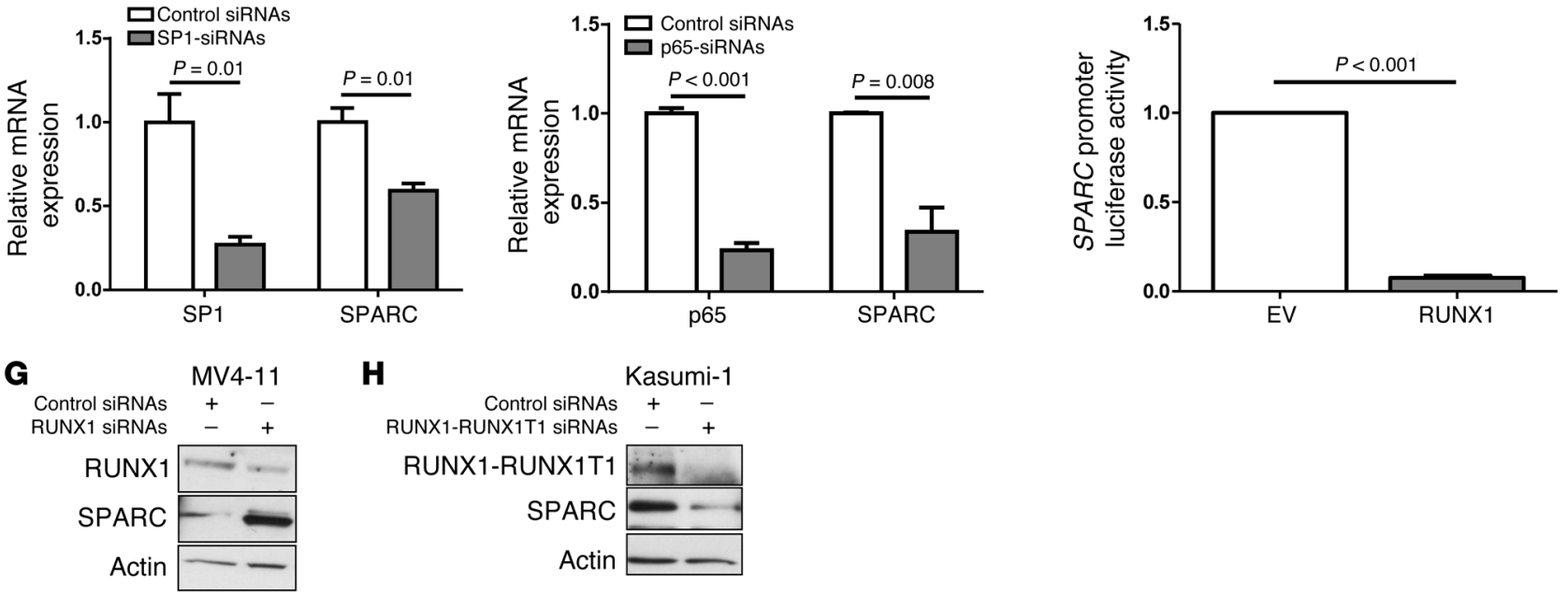

H

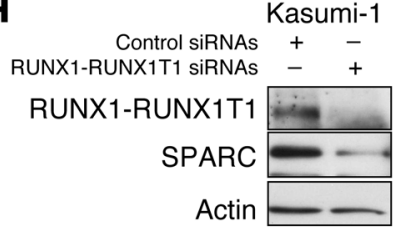

I

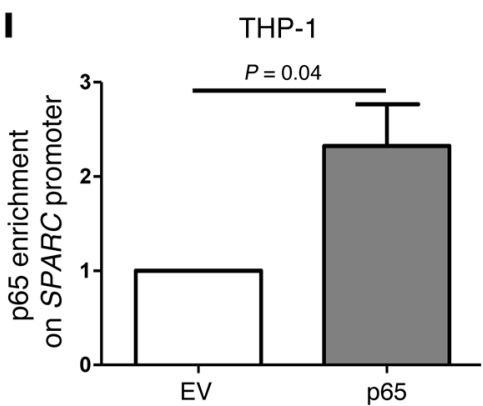

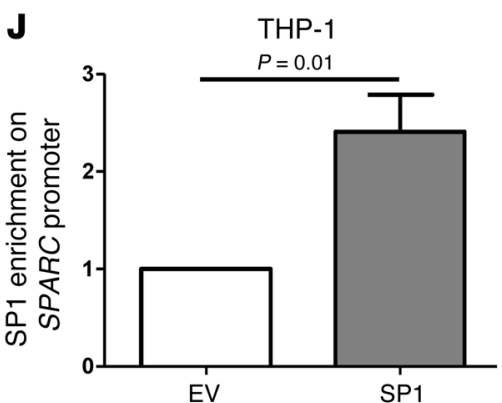

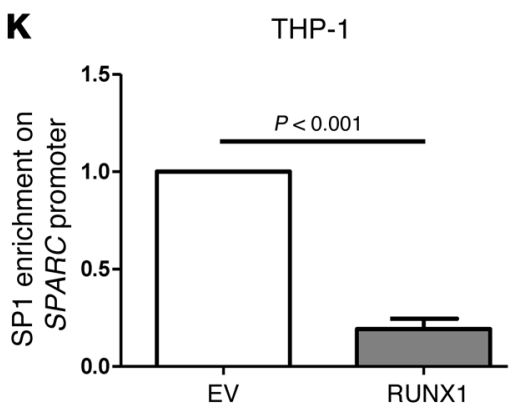

Figure 7

SP1, NF-кB, and miR-29b modulate SPARC expression in AML. (A) Luciferase activities measured in 293T cells cotransfected with PGL4.11 luciferase vector containing the SPARC promoter region (PGL4.11/SPARC promoter) and either SP1-expressing (left panel) or p65-expressing (right panel) vectors; measurements were normalized to that obtained in 293T cells cotransfected with PGL4.11/SPARC-promoter vector or EV. (B) Western blot analysis for SPARC protein expression measured in THP-1 cells transfected with SP1-expressing vector or (C) p65-expressing vector. (D) SPARC mRNA expression measured in Kasumi-1 cells transfected with SP1 siRNAs or (E) p65 siRNAs. (F) Luciferase activities measured in 293T cells cotransfected with a PGL4.11 luciferase vector containing the SPARC promoter region (PGL4.11/SPARC-promoter) and a RUNX1-expressing vector; measurements were normalized to that in 293T cells cotransfected with PGL4.11/SPARC-promoter vector and EV. (G) Western blot analysis showing SPARC protein levels in MV4-11 cells transfected with RUNX1 siRNAs or (H) Kasumi-1 cells transfected with RUNX1-RUNX1T1 siRNA. (I) ChIP showed p65 enrichment on SPARC promoter in THP-1 cells transfected with p65-expressing vector. (J) ChIP showed SP1 enrichment on SPARC promoter in THP-1 cells transfected with SP1-expressing vector. (K) Change in SP1 enrichment on SPARC promoter in RUNX1-transfected THP-1 cells compared with EV-transfected cells. Data represent mean \pm SEM.

the protein interacted with the leukemia cell membrane integrins and activated ILK/AKT $/ \beta$-catenin signaling, thereby promoting cell engraftment, growth, and survival. Pharmacologic disruption of the SP1/NF- $\mathrm{KB}$ transactivation complex $(45,62)$ resulted in SPARC downregulation, thereby representing a potentially novel therapeutic strategy for SPARC-dependent AML.
A finding in our study was that SPARC enhanced $\beta$-catenin activity in AML cells, known to be required for leukemia growth and stemness. The increase in $\beta$-catenin activity was seemingly enhanced via the SPARC-integrin-ILK axis. ILK is a kinase that links the cell adhesion receptors, integrins, and growth factors with the downstream signaling pathways involving AKT and 
A

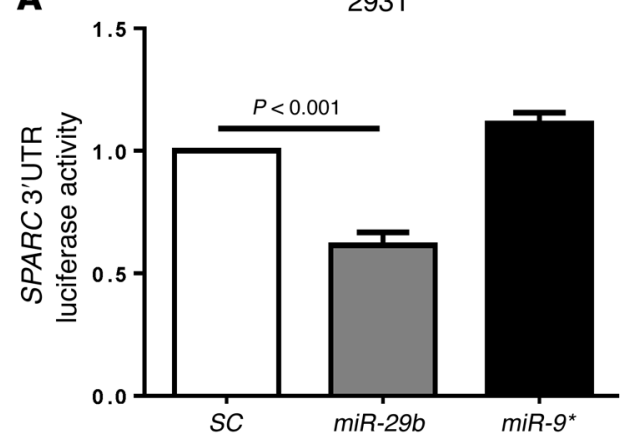

D

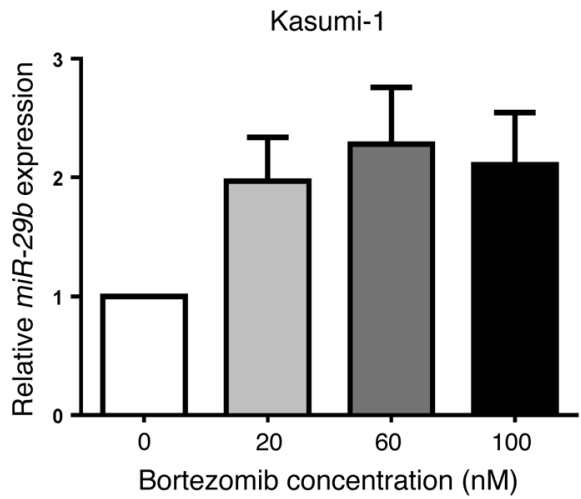

B

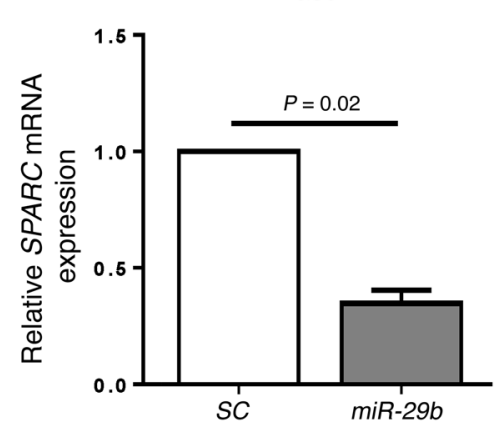

C

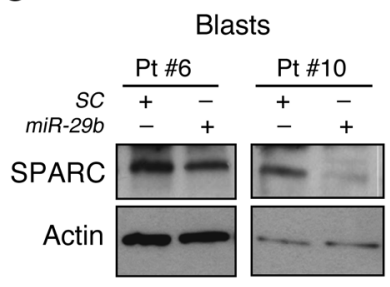

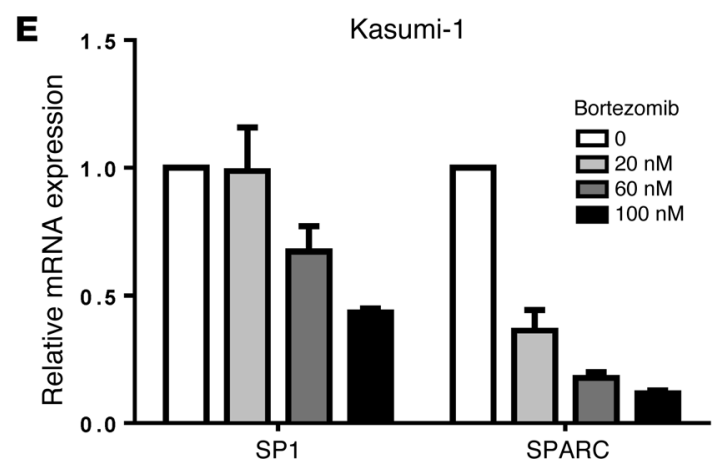

F

Kasumi-1

$\begin{array}{lllll}\text { Bortezomib (nM) } & 0 & 20 & 60 & 100\end{array}$

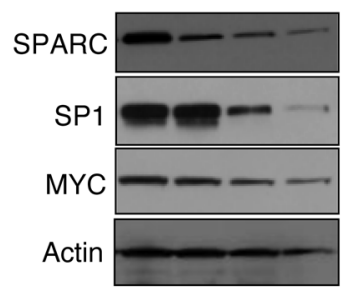

G

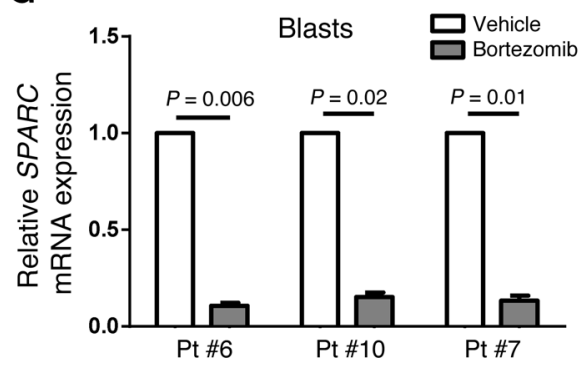

H

Blasts

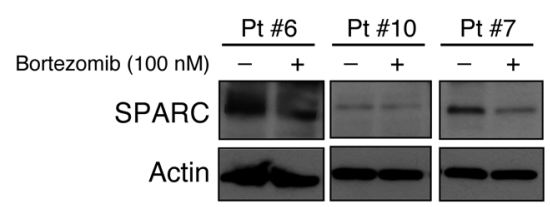

Figure 8

Targeting SPARC expression in AML. (A) SPARC 3' UTR-luciferase activity in the presence of miR-29b was measured by cotransfecting 293 T cells with SPARC-3' UTR reporter and a synthetic microRNA; values were normalized to those of cells transfected with scramble. (B) Ectopic expression of synthetic miR-29b significantly decreases SPARC mRNA expression in Kasumi-1 cells. (C) SPARC protein in AML blast samples (patients 6 and 10) at 24 hours following transfection with miR-29b. (D) miR-29b levels, (E) mRNA levels of SPARC and SP1, and (F) Western blot analysis showing SPARC, SP1, and MYC protein levels, measured in Kasumi-1 cells treated with 20, 60, and $100 \mathrm{nM}$ of bortezomib. (G) SPARC mRNA and $(\mathbf{H})$ protein levels in blasts from 3 primary AML samples (patients 6, 10, and 7) treated with $100 \mathrm{nM}$ bortezomib and assessed 24 hours later.

GSK3 $\beta$ (43). This ultimately results in stabilization and nuclear localization of $\beta$-catenin and activation of genes promoting cellcycle progression and proliferation (63). Consistent with these results, we observed that SPARC levels were higher in both normal and immature AML cell subpopulations that indeed displayed a higher engraftment potential in immunodeficient mice. While SPARC/ILK/AKT signaling was found involved in glioma cell invasion and survival (64), to our knowledge, the relevance of this mechanism in AML has not been previously reported.

Having observed that SPARC overexpression contributes to more aggressive phenotype in AML patients, we asked whether it is possible to target SPARC expression and demonstrate the clinical relevance of our findings. We showed that, similarly to other oncogenes, constitutive SPARC expression was dependent on a previously reported regulatory molecular network that involves SP1/NF-KB and miR-29b (45). The leukemogenic role of this network that can be targeted pharmacologically has been extensively studied by our group not only in AML (45) but also in other types of leukemia $(45,62)$. Our data support the view that SPARC overexpression is a potentially actionable therapeutic target via pharmacological disruption of the SP1/NF- $\mathrm{KB} / m i R-29 b$ network by, for example, bortezomib, used here as a proof of concept. Other compounds, including inhibitors of HDAC (e.g., vorinostat, AR-42) or SP1 (e.g., mithramycin A) or synthetic miR-29b mimics may also interfere with the SP1/NF- $\mathrm{BB} / m i R-29 b$ network and downregulate SPARC $(45,50,65,66)$. 
While our findings emphasize a role of SPARC in the homeostasis of myeloblasts, several other reports have underscored the interplay of SPARC protein with the microenvironment to create a niche favorable for cancer growth (8). Thus, future studies of SPARC in AML need to address the dual intracellular and extracellular role of this protein.

In conclusion, SPARC deregulation is clinically relevant in AML because overexpression of this gene independently predicts adverse outcome in subsets of AML patients, contributes to aggressive AML growth likely via $\beta$-catenin activation, and is an actionable therapeutic target. This suggests that future clinical studies targeting the activity of SPARC protein are warranted in AML.

\section{Methods}

Plasmids and reagents. SPARC clone was purchased from Invitrogen and inserted in pLenti6.2/V5-DEST (Invitrogen) and MIGR (Addgene). pCMVp65, pSUPER-SP1, and PCMV-RUNX1 expression vectors were also used (45). OFF-TARGET control and ON-TARGET plus siRNA-SMARTpool reagents against SPARC, SP1, p65, and RUNX1 (each contains a mix of at least 4 different sequences) were purchased from Dharmacon. siRNA against RUNX1-RUNX1T1 was custom designed (Dharmacon). SPARC shRNA (a set of 6 different sequences) was purchased from Thermo Fisher Scientific. Bortezomib is commercially available (Millennium Pharmaceuticals).

Cell lines and primary blasts. THP-1, MV4-11, Kasumi-1, and CG-SH cells were cultured in RPMI medium supplemented with $10 \%-20 \%$ FBS. Blasts from AML patients were maintained in RPMI medium supplemented with $20 \%$ FBS and $1 \times$ StemSpan CC100 (StemCell Technologies). AML blasts used in the experiments were obtained from apheresis blood samples collected from patients treated at OSU and stored in the OSU Leukemia Tissue Bank.

Transient transfection, RNA interference, and viral induction. Transient transfection of cells was performed utilizing 1 to $2 \mu$ g of plasmid or $1 \mathrm{nmol}$ of siRNA per reaction and Nucleofector Kit (Amaxa) according to the manufacturer's instructions (67). Retroviral and lentiviral infection were performed with an MOI of 3 and greater than 10 , respectively, to obtain efficiencies that approximately reached $40 \%$, as previously reported $(68,69)$. Cells were selected by antibiotic selection with blasticidin or sorting for GFP-positive cells.

RNA extraction and RNA expression quantification. Total RNA was extracted using TrizoL reagent (Invitrogen). SPARC expression in CN-AML patients was measured by Nano-String nCounter system according to the manufacturer's instructions (Nano-String). Gene cDNA was synthesized using SuperScript III reagents (Invitrogen) and the TaqMan MicroRNA Reverse Transcription Kit (Applied Biosystems) according to the manufacturer's instructions. Quantitative real-time RT-PCR (qRT-PCR) was performed using commercially available TaqMan Gene Expression Assay primers and probes and the 7900HT Fast Real-Time PCR System (Applied Biosystems). The expression levels were normalized to $18 \mathrm{~S}$ for gene or $\mathrm{U} 44$ for microRNA expression.

Immunoprecipitation, Western blot analysis, ChIP, and antibodies. Immunoprecipitation and Western blot were performed as previously described (70). ChIP assays were performed using the EZ ChIP Kit (Millipore) according to the manufacturer's protocol. DNA was quantified using qRT-PCR with SYBR green incorporation (Applied Biosystems) and primers specific for SPARC promoter and normalized to their input. Antibodies and primers are listed in Supplemental Experimental Procedures.

Clonogenic and viability analyses. Methylcellulose clonogenic assays were carried out by plating $1 \times 10^{3}$ cells of different cell lines or $2 \times 10^{4}$ primary blasts in $0.9 \%$ MethoCult (StemCell Technologies) (68). Colony numbers were scored 10 days later.

For viability and apoptosis analysis, cells were washed with PBS and resuspended in $50 \mu \mathrm{l}$ binding buffer containing $2 \mu \mathrm{l}$ of annexin $\mathrm{V}$ (eBioscience) and $5 \mu \mathrm{l}$ propidium iodide (PI) (eBioscience). After 20 minutes incubation, fluorescence was quantified by flow cytometry on a FACSCalibur instrument.

Immunofluorescent staining, cell sorting, flow cytometry and confocal microscopy. Cells were washed and stained with CD34, CD38, CD45RA, and CD123 antibodies (eBioscience) and sorted into hematopoietic stem cells and HSC/ $\mathrm{MPP}\left(\mathrm{CD} 34^{+} / \mathrm{CD} 38^{-}\right), \mathrm{CMP}\left(\mathrm{CD} 34^{+} / \mathrm{CD}^{2} 5 \mathrm{RA}^{-} / \mathrm{CD} 123^{\mathrm{lo}}\right)$ and $\mathrm{GMP}\left(\mathrm{CD} 34^{+} /\right.$ $\mathrm{CD}^{2} 5 \mathrm{RA}^{+} / \mathrm{CD} 123^{\mathrm{lo}}$ ) using BD FACSAriaII cell sorter (BD Biosciences).

For confocal microscopy analysis, $1 \times 10^{6}$ cells were processed as detailed in Supplemental Experimental Procedures.

In vivo experiments. Four- to six-week-old NOD/SCID mice (The Jackson Laboratory) were i.v. injected via tail vein with $5 \times 10^{6} \mathrm{THP}-1$ cells infected with plenti-EV or plenti-SPARC. Eight weeks later, mice $(n=6)$ were euthanized; spleens, livers, and sternums were isolated. Xenograft transplantations were performed as detailed in Supplemental Experimental Procedures.

Patients. We studied pretreatment bone marrow and blood samples with $20 \%$ or more blasts from 153 patients with primary CN-AML (age range, $18-59$ years) who were treated with cytarabine-daunorubicin-based regimens on Cancer and Leukemia Group B (CALGB) 9621 or 19808 protocols (71). Per protocol, no patient included in our analysis received allogeneic transplantation in first CR. The median follow-up was 8.7 years.

Cytogenetic and mutational analyses. The diagnosis of CN-AML was based on the analysis of 20 or more metaphases in pretreatment bone marrow specimens subjected to short-term cultures and confirmed by central karyotype review (72). Patients were also characterized centrally for FLT3-ITD (73), FLT3 tyrosine kinase domain (TKD) mutations (74), MLL partial tandem duplication (75, 76), NPM1 (28, 77), WT1 (78), CEBPA (79), IDH1, and IDH2 (26) TET2 (80), ASXL1 (81), and DNMT3A (71) mutations as previously reported.

Statistics. Statistical analyses relative to clinical outcome were performed by the Alliance Statistics and Data Center. Definitions of clinical end points $-\mathrm{CR}, \mathrm{DFS}$, and OS - are as reported previously (26). The differences in baseline clinical and molecular features between higher and lower SPARC expressers were tested using the Fisher's exact and Wilcoxon rank sum tests for categorical and continuous variables, respectively. Estimated probabilities of DFS and OS were calculated using the Kaplan-Meier method, and the logrank test evaluated differences between survival distributions. Mechanistic and biological experiments were analyzed with paired and unpaired 2-tailed $t$ tests as required, and a $P$ value of less than 0.05 was considered significant. Data are presented as mean \pm SEM in all figures where error bars are shown.

Study approval. Informed consent to use patient samples for investigational studies was obtained from each patient according to OSU institutional guidelines and in accordance with the Declaration of Helsinki, and protocols were approved by the IRB at OSU and each center involved in the CALGB trials. All animal studies were performed in accordance with OSU institutional guidelines for animal care and under protocols approved by the OSU Institutional Animal Care and Use Committee.

\section{Acknowledgments}

This work was supported in part by grants from the National Cancer Institute (Bethesda, Maryland, USA) (CA101140, CA016058, CA77658, CA55164, CA100632, and CA140158), the Coleman Leukemia Research Foundation, and the Conquer Cancer Foundation (to J.H. Mendler).

Received for publication May 14, 2013, and accepted in revised form January 2, 2014.

Address correspondence to: Guido Marcucci, The Ohio State University Comprehensive Cancer Center, Biomedical Research Tower, 460 W. 12th Ave, Columbus, Ohio 43210, USA. Phone: 614.366.2261; Fax: 614.688.4028; E-mail: guido.marcucci@osumc.edu. 
1. Swaroop A, Hogan BL, Francke U. Molecular analysis of the cDNA for human SPARC/osteonectin/BM-40: sequence, expression, and localization of the gene to chromosome 5q31-q33. Genomics. 1988;2(1):37-47.

2. Lane TF, Sage EH. The biology of SPARC, a protein that modulates cell-matrix interactions. FASEB J. 1994;8(2):163-173.

3. Motamed K. SPARC (osteonectin/BM-40). Int J Biochem Cell Biol. 1999;31(12):1363-1366.

4. Kupprion C, Motamed K, Sage EH. SPARC (BM40 , osteonectin) inhibits the mitogenic effect of vascular endothelial growth factor on microvascular endothelial cells. J Biol Chem. 1998; 273(45):29635-29640.

5. Phan E, Ahluwalia A, Tarnawski AS. Role of SPARC - matricellular protein in pathophysiology and tissue injury healing. Implications for gastritis and gastric ulcers. Med Sci Monit. 2007;13(2):RA25-RA30.

6. Raines EW, Lane TF, Iruela-Arispe ML, Ross R, Sage $\mathrm{EH}$. The extracellular glycoprotein SPARC interacts with platelet-derived growth factor (PDGF)- $\mathrm{AB}$ and -BB and inhibits the binding of PDGF to its receptors. Proc Natl Acad Sci US A. 1992;89(4):1281-1285.

7. Schiemann BJ, Neil JR, Schiemann WP. SPARC inhibits epithelial cell proliferation in part through stimulation of the transforming growth factor-beta-signaling system. Mol Biol Cell. 2003; 14(10):3977-3988

8. Podhajcer OL, Benedetti LG, Girotti MR, Prada F, Salvatierra E, Llera AS. The role of the matricellular protein SPARC in the dynamic interaction between the tumor and the host. Cancer Metastasis Rev. 2008;27(4):691-705.

9. Mok SC, Chan WY, Wong KK, Muto MG, Berkowitz RS. SPARC, an extracellular matrix protein with tumor-suppressing activity in human ovarian epithelial cells. Oncogene. 1996;12(9):1895-1901.

10. Cheetham S, Tang MJ, Mesak F, Kennecke H, Owen D, Tai IT. SPARC promoter hypermethylation in colorectal cancers can be reversed by 5 -Aza2 'deoxycytidine to increase SPARC expression and improve therapy response. Br J Cancer. 2008; 98(11):1810-1819.

11. Yang E, Kang HJ, Koh KH, Rhee H, Kim NK, Kim $H$. Frequent inactivation of SPARC by promoter hypermethylation in colon cancers. Int $J$ Cancer. 2007;121(3):567-575

12. Sato $\mathrm{N}$, et al. SPARC/osteonectin is a frequent target for aberrant methylation in pancreatic adenocarcinoma and a mediator of tumor-stromal interactions. Oncogene. 2003;22(32):5021-5030.

13. Watkins G, Douglas-Jones A, Bryce R, Mansel RE, Jiang WG. Increased levels of SPARC (osteonectin) in human breast cancer tissues and its association with clinical outcomes. Prostaglandins Leukot Essent Fatty Acids. 2005;72(4):267-272.

14. Gilles C, Bassuk JA, Pulyaeva H, Sage EH, Foidart JM, Thompson EW. SPARC/osteonectin induces matrix metalloproteinase 2 activation in human breast cancer cell lines. Cancer Res. 1998;58(23):5529-5536.

15. Ledda F, Bravo AI, Adris S, Bover L, Mordoh J, Podhajcer OL. The expression of the secreted protein acidic and rich in cysteine (SPARC) is associated with the neoplastic progression of human melanoma. J Invest Dermatol. 1997;108(2):210-214.

16. Kato Y, et al. High production of SPARC/osteonectin/BM-40 in mouse metastatic B16 melanoma cell lines. Pathol Oncol Res. 2000;6(1):24-26.

17. Golembieski WA, Ge S, Nelson K, Mikkelsen T, Rempel SA. Increased SPARC expression promotes U87 glioblastoma invasion in vitro. Int J Dev Neurosci. 1999;17(5-6):463-472.

18. Koukourakis MI, et al. Enhanced expression of SPARC/osteonectin in the tumor-associated stroma of non-small cell lung cancer is correlated with markers of hypoxia/acidity and with poor prognosis of patients. Cancer Res. 2003;63(17):5376-5380.
19. Witkiewicz AK, et al. Stromal CD10 and SPARC expression in ductal carcinoma in situ (DCIS) patients predicts disease recurrence. Cancer Biol Ther. 2010;10(4):391-396.

20. Liang JF, et al. Relationship and prognostic significance of SPARC and VEGF protein expression in colon cancer. J Exp Clin Cancer Res. 2010;29:71.

21. Fidler C, Strickson A, Boultwood J, Waincoat JS. Mutation analysis of the SPARC gene in the 5q-syndrome. Am J Hematol. 2000;64(4):324.

22. Lehmann S, O'Kelly J, Raynaud S, Funk SE, Sage $\mathrm{EH}$, Koeffler HP. Common deleted genes in the 5q- syndrome: thrombocytopenia and reduced erythroid colony formation in SPARC null mice. Lenkemia. 2007;21(9):1931-1936.

23. Pellagatti A, et al. Lenalidomide inhibits the malignant clone and up-regulates the SPARC gene mapping to the commonly deleted region in 5qsyndrome patients. Proc Natl Acad Sci U S A. 2007; 104(27):11406-11411.

24. DiMartino JF, et al. Low or absent SPARC expression in acute myeloid leukemia with MLL rearrangements is associated with sensitivity to growth inhibition by exogenous SPARC protein. Leukemia. 2006;20(3):426-432.

25. Fenouille $\mathrm{N}$, et al. Persistent activation of the Fyn/ERK kinase signaling axis mediates imatinib resistance in chronic myelogenous leukemia cells through upregulation of intracellular SPARC. Cancer Res. 2010;70(23):9659-9670.

26. Marcucci G, et al. IDH1 and IDH2 gene mutations identify novel molecular subsets within de novo cytogenetically normal acute myeloid leukemia: a Cancer and Leukemia Group B study. J Clin Oncol. 2010;28(14):2348-2355

27. Schwind S, et al. BAALC and ERG expression levels are associated with outcome and distinct gene and microRNA expression profiles in older patients with de novo cytogenetically normal acute myeloid leukemia: a Cancer and Leukemia Group B study. Blood. 2010;116(25):5660-5669.

28. Becker H, et al. Favorable prognostic impact of NPM1 mutations in older patients with cytogenetically normal de novo acute myeloid leukemia and associated gene- and microRNA-expression signatures: a Cancer and Leukemia Group B study. JClin Oncol. 2010;28(4):596-604.

29. Payton JE, et al. High throughput digital quantification of mRNA abundance in primary human acute myeloid leukemia samples. J Clin Invest 2009; 119(6):1714-1726.

30. Sturm RA, et al. Osteonectin/SPARC induction by ectopic beta(3) integrin in human radial growth phase primary melanoma cells. Cancer Res. 2002; 62(1):226-232.

31. Pavasant P, Yongchaitrakul T. Secreted protein acidic, rich in cysteine induces pulp cell migration via $\alpha v \beta 3$ integrin and extracellular signal-regulated kinase. Oral Dis. 2008;14(4):335-340.

32. McDonald PC, Fielding AB, Dedhar S. Integrinlinked kinase - essential roles in physiology and cancer biology. J Cell Sci. 2008;121(pt 19):3121-3132.

33. Barker TH, et al. SPARC regulates extracellular matrix organization through its modulation of integrin-linked kinase activity. J Biol Chem. 2005; 280(43):36483-36493.

34. Weaver MS, Workman G, Sage EH. The copper binding domain of SPARC mediates cell survival in vitro via interaction with integrin beta 1 and activation of integrin-linked kinase. J Biol Chem 2008; 283(33):22826-22837.

35. Novak A, Dedhar S. Signaling through beta-catenin and Lef/Tcf. Cell Mol Life Sci. 1999;56(5-6):523-537.

36. He XC, et al. PTEN-deficient intestinal stem cells initiate intestinal polyposis. Nat Genet. 2007; 39(2):189-198.

37. Siapati EK, et al. Proliferation and bone marrow engraftment of AML blasts is dependent on beta-catenin signalling. Br J Haematol. 2011; 152(2):164-174.

38. Wang Y, et al. The Wnt/beta-catenin pathway is required for the development of leukemia stem cells in AML. Science. 2010;327(5973):1650-1653.

39. Shi Q, et al. Secreted protein acidic, rich in cysteine (SPARC), mediates cellular survival of gliomas through AKT activation. J Biol Chem. 2004; 279(50):52200-52209.

40. Nie J, Sage EH. SPARC inhibits adipogenesis by its enhancement of beta-catenin signaling. J Biol Chem. 2009;284(2):1279-1290.

41. Chang W, Wei K, Jacobs SS, Upadhyay D, Weill D, Rosen GD. SPARC suppresses apoptosis of idiopathic pulmonary fibrosis fibroblasts through constitutive activation of $\beta$-catenin. J Biol Chem. 2010;285(11):8196-8206.

42. Lee SL, et al. Functional role of mTORC2 versus integrin-linked kinase in mediating Ser473-Akt phosphorylation in PTEN-negative prostate and breast cancer cell lines. PLoS One. 2013;8(6):e67149.

43. Tabe $Y$, et al. Activation of integrin-linked kinase is a critical prosurvival pathway induced in leukemic cells by bone marrow-derived stromal cells. Cancer Res. 2007;67(2):684-694

44. Zhu XC, et al. microRNA-29a suppresses cell proliferation by targeting SPARC in hepatocellular carcinoma. Int J Mol Med. 2012;30(6):1321-1326.

45. Liu S, et al. Sp1/NFkB/HDAC/miR-29b regulatory network in KIT-driven myeloid leukemia. Cancer Cell. 2010;17(4):333-347.

46. Luna C, Li G, Qiu J, Epstein DL, Gonzalez P. Role of miR-29b on the regulation of the extracellular matrix in human trabecular meshwork cells under chronic oxidative stress. Mol Vis. 2009;15:2488-2497.

47. Kapinas K, Kessler CB, Delany AM. miR-29 suppression of osteonectin in osteoblasts: regulation during differentiation and by canonical Wnt signaling. J Cell Biochem. 2009;108(1):216-224.

48. Garzon R, et al. MicroRNA-29b induces global DNA hypomethylation and tumor suppressor gene reexpression in acute myeloid leukemia by targeting directly DNMT3A and 3B and indirectly DNMT1. Blood. 2009;113(25):6411-6418.

49. Bernot KM, et al. Eradicating acute myeloid leukemia in a MllPTD/wt:Flt3ITD/wt murine model: a path to novel therapeutic approaches for human disease. Blood. 2013;122(23):3778-3783.

50 . Blum W, et al. Clinical and pharmacodynamic activity of bortezomib and decitabine in acute myeloid leukemia. Blood. 2012;119(25):6025-6031.

51. Porter PL, Sage EH, Lane TF, Funk SE, Gown AM. Distribution of SPARC in normal and neoplastic human tissue. J Histochem Cytochem. 1995; 43(8):791-800.

52. Massi D, Franchi A, Borgognoni L, Reali UM, Santucci M. Osteonectin expression correlates with clinical outcome in thin cutaneous malignant melanomas. Hum Pathol 1999;30(3):339-344.

53. McClung HM, Golembieski WA, Schultz CR, Jankowski M, Schultz LR, Rempel SA. Deletion of the SPARC acidic domain or EGF-like module reduces SPARC-induced migration and signaling through p38 MAPK/HSP27 in glioma. Carcinogenesis. 2012;33(2):275-284.

54. Golembieski WA, et al. HSP27 mediates SPARCinduced changes in glioma morphology, migration, and invasion. Glia. 2008;56(10):1061-1075.

55. Yunker CK, et al. SPARC-induced increase in glioma matrix and decrease in vascularity are associated with reduced VEGF expression and secretion. Int J Cancer. 2008;122(12):2735-2743.

56. Ledda MF, et al. Suppression of SPARC expression by antisense RNA abrogates the tumorigenicity of human melanoma cells. Nat Med. 1997;3(2):171-176.

57. Yiu GK, et al. SPARC (secreted protein acidic and rich in cysteine) induces apoptosis in ovarian cancer cells. Am J Pathol. 2001;159(2):609-622. 
58. Aoi W, et al. A novel myokine, secreted protein acidic and rich in cysteine (SPARC), suppresses colon tumorigenesis via regular exercise. Gut. 2012; 62(6):882-889.

59. Siva K, Jaako P, Miharada K. SPARC is dispensable for murine hematopoiesis, despite its suspected pathophysiological role in $5 \mathrm{q}$ - myelodysplastic syndrome. Leukemia. 2012;26(11):2416-2419.

60. Tripodo C, et al. Stromal SPARC contributes to the detrimental fibrotic changes associated with myeloproliferation while its deficiency favors myeloid cell expansion. Blood. 2012;120(17):3541-3554.

61. Guzman ML, et al. Preferential induction of apoptosis for primary human leukemic stem cells. Proc Natl Acad Sci U S A. 2002;99(25):16220-16225.

62. Mishra A, et al. Aberrant overexpression of IL-15 initiates large granular lymphocyte leukemia through chromosomal instability and DNA hypermethylation. Cancer Cell. 2012;22(5):645-655.

63. Hannigan G, Troussard AA, Dedhar S. Integrinlinked kinase: a cancer therapeutic target unique among its ILK. Nat Rev Cancer. 2005;5(1):51-63.

64. Shi Q, et al. Targeting SPARC expression decreases glioma cellular survival and invasion associated with reduced activities of FAK and ILK kinases. Oncogene. 2007;26(28):4084-4094.

65. Mims A, et al. Increased anti-leukemic activity of decitabine via AR-42-induced upregulation of miR29b: a novel epigenetic-targeting approach in acute myeloid leukemia. Leukemia. 2013;27(4):871-878.

66. Huang X, et al. Targeted delivery of microRNA-29b by transferrin-conjugated anionic lipopolyplex nanoparticles: a novel therapeutic strategy in acute myeloid leukemia. Clin Cancer Res. 2013;19(9):2355-2367.
67. Hickey CJ, et al. Lenalidomide-mediated enhanced translation of $\mathrm{C} / \mathrm{EBP} \alpha$-p30 protein up-regulates expression of the antileukemic microRNA-181a in acute myeloid leukemia. Blood. 2013;121(1):159-169.

68. Eiring AM, et al. miR-328 functions as an RNA decoy to modulate hnRNP E2 regulation of mRNA translation in leukemic blasts. Cell. 2010;140(5):652-665.

69. Dorrance AM, et al. The Rac GTPase effector p 21 activated kinase is essential for hematopoietic stem/progenitor cell migration and engraftment. Blood. 2013;121(13):2474-2482.

70. Liu S, et al. Bortezomib induces DNA hypomethylation and silenced gene transcription by interfering with $\mathrm{Sp} 1 / \mathrm{NF}-\kappa \mathrm{B}-$ dependent DNA methyltransferase activity in acute myeloid leukemia. Blood. 2008;111(4):2364-2373.

71. Marcucci G, et al. Age-related prognostic impact of different types of DNMT3A mutations in adults with primary cytogenetically normal acute myeloid leukemia. J Clin Oncol. 2012;30(7):742-750.

72. Mrozek K, et al. Central review of cytogenetics is necessary for cooperative group correlative and clinical studies of adult acute leukemia: the Cancer and Leukemia Group B experience. Int J Oncol. 2008;33(2):239-244.

73. Thiede C, et al. Analysis of FLT3-activating mutations in 979 patients with acute myelogenous leukemia: association with FAB subtypes and identification of subgroups with poor prognosis. Blood. 2002;99(12):4326-4335

74. Whitman SP, et al. FLT3 D835/I836 mutations are associated with poor disease-free survival and a distinct gene-expression signature among younger adults with de novo cytogenetically normal acute myeloid leukemia lacking FLT3 internal tandem duplications. Blood. 2008;111(3):1552-1559.

75. Whitman SP, et al. Long-term disease-free survivors with cytogenetically normal acute myeloid leukemia and MLL partial tandem duplication: a Cancer and Leukemia Group B study. Blood. 2007; 109(12):5164-5167.

76. Caligiuri MA, et al. Partial tandem duplication of ALL1 as a recurrent molecular defect in acute myeloid leukemia with trisomy 11. Cancer Res. 1996; 56(6):1418-1425.

77. Dohner K, et al. Mutant nucleophosmin (NPM1) predicts favorable prognosis in younger adults with acute myeloid leukemia and normal cytogenetics: interaction with other gene mutations. Blood. 2005;106(12):3740-3746.

78. Becker H, et al. Mutations of the Wilms tumor 1 gene (WT1) in older patients with primary cytogenetically normal acute myeloid leukemia: a Cancer and Leukemia Group B study. Blood. 2010;116(5):788-792.

79. Marcucci G, et al. Prognostic significance of, and gene and microRNA expression signatures associated with, CEBPA mutations in cytogenetically normal acute myeloid leukemia with high-risk molecular features: a Cancer and Leukemia Group B Study. J Clin Oncol. 2008;26(31):5078-5087.

80. Metzeler KH, et al. TET2 mutations improve the new European LeukemiaNet risk classification of acute myeloid leukemia: a Cancer and Leukemia Group B study. J Clin Oncol. 2011;29(10):1373-1381.

81. Metzeler KH, et al. ASXL1 mutations identify a highrisk subgroup of older patients with primary cytogenetically normal AML within the ELN Favorable genetic category. Blood. 2011;118(26):6920-6929. 